THE ASTROPHYSICAL JouRNAL SUPPLEMENT SERIES, 88:357-381, 1993 October

(c) 1993. The American Astronomical Society. All rights reserved. Printed in U.S.A.

\title{
DOUBLE TROUGHS IN BROAD ABSORPTION LINE QUASARS AND Ly $\alpha-N$ v LINE-LOCKING ${ }^{1}$
}

\author{
KIRK T. KORISTA ${ }^{2}$ \\ Observatories of the Carnegie Institution of Washington, 813 Santa Barbara Street, Pasadena, CA 91101 \\ G. MARK VOIT \\ California Institute of Technology, Theoretical Astrophysics, Mail Code 130-33, Pasadena, CA 91125 \\ AND \\ SIMON L. MORRIS ${ }^{3}$ AND RAY J. WEYMANN \\ Observatories of the Carnegie Institution of Washington, 813 Santa Barbara Street, Pasadena, CA 91101 \\ Received 1992 September 15; accepted 1993 March 17
}

\begin{abstract}
In a previous comparative study by Weymann et al. of the emission line and continuum properties of radioquiet QSOs and broad absorption-line (BAL) QSOs, a difference spectrum, in the region of the C IV $\lambda 1549$ trough, comprising the mean spectrum of 34 BALQSOs and the mean spectrum of 31 non-BALQSOs, surprisingly revealed double troughs separated in velocity space by the $\mathrm{N} v \lambda 1240-\mathrm{Ly} \alpha$ splitting of $\sim 5900 \mathrm{~km} \mathrm{~s}^{-1}$. In this contribution we investigate the reality of these features in a spectroscopic sample of 72 BALQSOs consisting of the original 34 BALQSOs and an additional 38 BALQSOs. An atlas of the C IV BALs of 72 BALQSOs is presented. We find that only $22 \%$ of this sample explicitly exhibits the double trough feature. When present the double troughs are deep. A Monte Carlo simulation of the mean C IV BAL suggests that the double-trough feature is real at only the $95 \%-98 \%$ level (as opposed to being the result of statistical fluctuations of BAL troughs occurring over random outflow velocities in a limited sample). We describe possible scenarios for their formation, involving the line-locking of $\mathrm{Ly} \alpha-\mathrm{N} v$ within the BAL region. We show that no simple mechanism involving radiation pressure alone can create the deep double troughs through the shadowing of $N \mathrm{v} \lambda 1240$ by Ly $\alpha$. More complicated amplification mechanisms may be necessary. If real, the double-trough signature has the following important implications to our understanding of the BAL phenomenon: (1) The occurrence of broad absorption is somehow tied to an absolute velocity frame which is the same from quasar to quasar. (2) It follows then that the outflowing material within the BAL region is accelerating. (3) If line-locking is the mechanism behind the formation of the double troughs, then radiation pressure plays an important role in the dynamics of the BAL region.
\end{abstract}

Subject headings: atomic processes - quasars: general

\section{INTRODUCTION}

Broad absorption-line QSOs (BALQSOs) are radio-quiet quasars (Stocke et al. 1992) which exhibit broad, blueshifted absorption troughs which can extend to outflow velocities of $0.1-0.2 c$. Korista et al. (1992) identified twelve or more ions in broad absorption between $700-1550 \AA$ in the rest-frame of the BALQSO 0226-1024. These ranged in ionization from $\mathrm{C}$ III to O VI. A small percentage of BALQSOs also show lowionization broad absorption due to Al III $\lambda 1858$ and/or $\mathrm{Mg}$ II $\lambda 2798$, in addition to the high-ionization troughs. In the most recent and complete comparison of the emission line and continuum properties between BALQSOs and non-BAL radio-quiet QSOs, Weymann et al. (1991) concluded that these two classes of quasars belong to the same population viewed at different aspect angles. If this is true, then all radio-quiet QSOs are propelling several solar masses of material per year outward at thousands to tens of thousands of kilometers per sec-

\footnotetext{
${ }^{1}$ Some of the observations reported here were made at Palomar Observatory as part of a collaborative agreement between the California Institute of Technology and the Carnegie Institution of Washington.

${ }_{2}^{2}$ Postal address: Space Telescope Science Institute, 3700 San Martin Drive, Baltimore, MD 21218.

${ }^{3}$ Postal address: Institute of Astronomy, Madingley Road, Cambridge CB3 OHA, UK.
}

ond. Recently proposed dynamical mechanisms for the absorbing outflows include the time-variable wind model of Braun \& Milgrom (1990) and the cosmic ray driven wind model of Begelman et al. (1991).

One of the more surprising results of the Weymann et al. (1991, hereafter W91) study is shown in their Figure 5. A difference spectrum, in the region of the C IV $\lambda 1549$ trough, comprising the mean spectrum of the BALQSOs and the mean spectrum of the non-BALQSOs, shows two distinct and localized minima separated by roughly the velocity splitting of $\mathrm{N} \mathrm{v}$ $\lambda 1240$ and $\operatorname{Ly} \alpha\left(\sim 5900 \mathrm{~km} \mathrm{~s}^{-1}\right)$ located near -4700 and $-10,500 \mathrm{~km} \mathrm{~s}^{-1}$. Henceforth, we refer to these together as the "double troughs", or separately as subtroughs 1 and 2, respectively. All velocities associated with the C IV BAL are measured with respect to the stronger line of the $C$ IV doublet, $\lambda 1548.2$, and are calculated with the relativistic correction. It was surprising that such structure had not "washed out" in the mean spectrum. A possible connection between BAL trough structure and the $\mathrm{Ly} \alpha-\mathrm{N} v$ splitting was first suggested by Turnshek et al. (1988), in a medium-high resolution spectroscopic study of three BALQSOs with P Cygni type absorption profiles (PHL 5200, RS 23, and 1413+1143). Turnshek et al. and W91 suggested that this structure was evidence for the importance of $\operatorname{Ly} \alpha$ radiation pressure in the dynamics of the outflow. 
In this paper: (1) We will attempt to determine whether the double-trough structure reported by W91 is real or is simply due to statistical fluctuations of BAL trough structure in a limited sample. (2) We investigate possible scenarios for the origin of the double troughs involving $\mathrm{Ly} \alpha-\mathrm{N} v$ line-locking.

We describe the BALQSO sample in $\S 2$ and the determination of the BALQSO redshifts in $\S 3$. The reality of the double troughs is investigated in $\S 4$, and we discuss line-locking scenarios for the formation of the double troughs in $\S 5$. A summary is provided in $\S 6$.

\section{THE BALQSO SPECTROSCOPIC SAMPLE}

In an effort to establish the reality of the double troughs seen in the mean BALQSO spectrum in W91, spectra of 32 more BALQSOs were obtained in addition to the 34 represented in Figure 5 of W91. These newly observed BALQSOs are tabulated in Table 1A, where we list their names, the observatory and instrument used, the exposure times, and the wavelength coverage. Adding six additional "low-ionization" BALQSOs which were not included in the difference spectrum of W91, brings the total number of BALQSOs for which we have C IV spectra to 72 . The entire sample is listed in column (1) of Table 1B. Partial spectra of the 32 additional BALQSOs, centered on the C IV BAL, are shown in Figure $1 a$. The BALQSOs which appeared in W91 are presented in Figure $1 b$ to more favorably show the C IV BALS in that sample. All spectra in Figures $1 a$ and $1 b$ have been resampled to $1 \AA$ per pixel (roughly $200 \mathrm{~km} \mathrm{~s}^{-1}$ at $1500 \AA$ ).

Nineteen new BALQSO spectra were obtained with the Double Spectrograph on the Hale $5 \mathrm{~m}$ telescope on Mount Palomar. W91 describe the data acquisition with this instrument in detail. Thirteen more new BALQSO spectra were obtained at the $2.5 \mathrm{~m}$ DuPont telescope of the Las Campanas Observatory (LCO) in 1991 October, using a Boller \& Chivens CCD spectrograph and a commercial Nikon $300 \mathrm{~mm}$ camera lens. For these data a Tektronix No. $21024 \times 1024$ ( 21 micron pixels) and a 1 ".5 slit (rotated to the parallactic angle) resulted in a spectral resolution of $2.19 \AA$ per pixel, 2.5 pixels FWHM. The spectral coverage was roughly $2200 \AA$. Two grating settings were employed to cover the $\mathrm{C}$ iv BAL trough from BALQSÖs at differing redshifts (3810-5990 and 4911-7109 $\AA$ ). The seeing was variable, the conditions were not photometric, and the objects were typically observed at air masses less than 1.2. The data were reduced in the usual fashion using IRAF. The data were put on a relative flux scale using flux standards from Stone \& Baldwin (1983). However, because of the defocusing and low throughput of the commercial camera at short wavelengths and unintended astigmatism in the system, most of the 1991 LCO observations suffered variable wavelength-dependent blue light loss. Some efforts were made to correct for this problem, but in general the relative fluxes shortward of $\sim 4300$ $\AA$ (roughly $1230-1500 \AA$ rest-frame) are uncertain by $\sim 25 \%-$ $50 \%$, with the relative fluxes at the shortest wavelengths the most uncertain. Fortunately, this problem had very little effect on the C IV BAL troughs in most of the Las Campanas sample.

\section{AN OBJECTIVE DETERMINATION OF THE REDSHIFTS}

Since Figure 5 in W91 suggests that BAL troughs occur at two particular outflow velocities in the QSO rest-frame in a
TABLE 1A

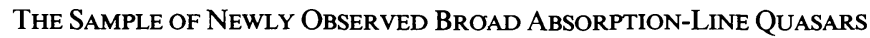

\begin{tabular}{|c|c|c|c|}
\hline $\begin{array}{l}\text { BALQSO Name } \\
\text { (1) }\end{array}$ & $\begin{array}{l}\text { Observatory/ } \\
\text { Instrument } \\
\text { (2) }\end{array}$ & $\begin{array}{c}\text { Exposure (s) } \\
\text { (3) }\end{array}$ & $\begin{array}{l}\text { Spectral } \\
\text { Coverage } \\
\text { (4) }\end{array}$ \\
\hline $0004+0147$ & PAL & 2400 & $3389-9863$ \\
\hline $0014-3913$ & LCO & 10800 & $3810-5990$ \\
\hline $0018+0047$ & PAL & 2400 & $3389-9863$ \\
\hline $0022+0150 \ldots \ldots$ & PAL & 2400 & 3389-9863 \\
\hline $0041-4023$ & LCO & 7200 & $3810-5990$ \\
\hline $0051-0019$ & PAL & 2400 & 3389-9863 \\
\hline $0054+0200$ & PAL & 2400 & $3389-9863$ \\
\hline $0105-2634$ & $\mathrm{LCO}$ & 5400 & $3810-5990$ \\
\hline $0113-4040 \ldots \ldots$ & LCO & 7200 & $4911-7109$ \\
\hline $0128-3647$ & LCO & 5400 & $3810-5990$ \\
\hline $0134-3740$ & LCO & 6300 & $3810-5990$ \\
\hline $0135-4001 \ldots \ldots$ & LCO & 7200 & $3810-5990$ \\
\hline $0254-3327 \ldots \ldots$ & LCO & 5400 & $3810-5990$ \\
\hline $0321-3344 \ldots \ldots$ & $\mathrm{LCO}$ & 4800 & $3810-5990$ \\
\hline $0324-4047 \ldots \ldots$ & $\mathrm{LCO}$ & 7050 & $4911-7109$ \\
\hline $0333-3801 \ldots \ldots$ & LCO & 5400 & $3810-5990$ \\
\hline $0340-4505 \ldots \ldots$ & LCO & 9600 & $3810-5990$ \\
\hline $0828+1229$ & PAL & 900 & $3189-9450$ \\
\hline $0842+3431 \ldots$ & PAL & 1800 & $3189-9450$ \\
\hline $0856+1714 \ldots$ & PAL & 3600 & $3189-9450$ \\
\hline $1009+0222 \ldots \ldots$ & PAL & 3600 & $3189-9450$ \\
\hline $1228+1216 \ldots \ldots$ & PAL & 3300 & $3189-9450$ \\
\hline $1336+1335 \ldots \ldots$ & PAL & 2400 & $3189-9450$ \\
\hline $1408+5642 \ldots$ & PAL & 1200 & $3189-9450$ \\
\hline $1414+0859$. & PAL & 1200 & $3189-9450$ \\
\hline $1423+5001 \ldots \ldots$ & PAL & 3600 & $3189-9450$ \\
\hline $1504+1041 \ldots \ldots$ & PAL & 1200 & $3189-9450$ \\
\hline $1524+5147 \ldots$ & PAL & 1200 & 3389-9863 \\
\hline $2202-2007 \ldots$ & PAL & 3600 & $3389-9863$ \\
\hline $2212-1759 \ldots$ & PAL & 2400 & $3389-9863$ \\
\hline $2240-3702 \ldots$ & LCO & 5400 & $3810-5990$ \\
\hline $2358+0216 \ldots \ldots$ & PAL & 2400 & 3389-9863 \\
\hline
\end{tabular}

a Observatory/instrument code: (PAL) Mt. Palomar $5 \mathrm{~m}$ Double Spectrograph; (LCO) Las Campanas $2.5 \mathrm{~m}$ Boller \& Chivens Spectrograph.

significant number of BALQSOs, it is important that we establish a systematic and objective method of estimating the BALQSO redshifts. The width of the double-trough features is $\sim 2500 \mathrm{~km} \mathrm{~s}^{-1}$; we must determine the redshifts to better than this value. Since Gaskell (1982), Corbin (1990), and Espey et al. (1989) have demonstrated that the redshifts of the C IV and $\mathrm{Mg}$ II emission-line peaks differ by as much as $1000-2000 \mathrm{~km}$ $\mathrm{s}^{-1}$ in some QSOs, it would be advantageous to include as many BAL-free broad emission lines as possible, in determining a mean redshift for the broad emission-line region. To determine each redshift correction we cross-correlated each BALQSO spectrum with a template using the FXCOR task within the NOAO.RV package of IRAF. We constructed the template by deredshifting each broad-emission line spectrum "by eye", subtracting a low-order polynomial fit to emissionline "free" regions (when available: near $1290 \AA$, 2000-2200 $\AA$, and near $3050 \AA$ ), and then combining all the spectra to create a high $\mathrm{S} / \mathrm{N}$ broad emission/absorption line mean spectrum of 72 BALQSOs.

After much trial and error, it was determined that whole spectra (excluding any BAL troughs) could not be used to derive reliable cross-correlation functions; the broad, low-contrast Fe II emission between 2200-2700 A generally gave spuri- 
TABLE 1B

ENTIRE C IV BROAD ABSORPTION-LINE QUASAR SAMPLE

\begin{tabular}{|c|c|c|c|c|c|c|c|}
\hline $\begin{array}{c}\text { BALQSO Name } \\
\text { (1) }\end{array}$ & $\begin{array}{c}z \\
(2)\end{array}$ & $\begin{array}{l}\text { Balnicity Index } \\
\left(\mathrm{km} \mathrm{s}^{-1}\right) \\
(3)\end{array}$ & $\begin{array}{c}\text { Double-Trough } \\
\text { Sample? } \\
\text { (4) }\end{array}$ & $\begin{array}{c}\text { BALQSO Name } \\
\text { (1) }\end{array}$ & $\begin{array}{c}z \\
(2)\end{array}$ & $\begin{array}{l}\text { Balnicity Index } \\
\left(\mathrm{km} \mathrm{s}^{-1}\right) \\
(3)\end{array}$ & $\begin{array}{c}\text { Double-Trough } \\
\text { Sample? } \\
\text { (4) }\end{array}$ \\
\hline $0004+0147 \ldots \ldots$ & 1.706 & 255 & & $1205+1436$ & 1.629 & 665 & \\
\hline $0014-3913 \ldots \ldots$ & 2.347 & 1725 & & $1208+1535 \ldots \ldots$ & 1.956 & 3813 & \\
\hline $0018+0047 \ldots \ldots$ & 1.816 & 329 & & $1212+1445 \ldots \ldots$ & 1.621 & 3438 & \\
\hline $0019+0107 \ldots \ldots$ & 2.124 & 2201 & & $1216+1103 \ldots \ldots$ & 1.615 & 4587 & yes \\
\hline $0021-0213 \ldots \ldots$ & 2.296 & 4607 & yes & $1228+1216 \ldots \ldots \ldots$ & 1.408 & 496 & \\
\hline $0022+0150 \ldots \ldots$ & 2.774 & 224 & yes & $1231+1320 \ldots \ldots$ & 2.386 & 1174 & yes \\
\hline $0025-0151 \ldots \ldots$ & 2.072 & 3436 & yes & $1232+1325 \ldots \ldots$ & 2.363 & 12448 & \\
\hline $0029+0017 \ldots \ldots$ & 2.226 & 4833 & & $1235+0857 \ldots \ldots$ & 2.885 & 276 & \\
\hline $0041-4023 \ldots \ldots$ & 2.476 & 3113 & & $1235+1453 \ldots \ldots$ & 2.694 & 2722 & \\
\hline $0043+0048 \ldots \ldots$ & 2.141 & 3822 & & $1239+0955 \ldots \ldots$ & 2.014 & 708 & \\
\hline $0051-0019 \ldots \ldots$ & 1.705 & 3244 & yes & $1240+1607 \ldots \ldots$ & 2.361 & 3344 & yes \\
\hline $0054+0200 \ldots \ldots$ & 1.868 & 498 & yes & $1243+0121 \ldots \ldots$ & 2.795 & 6243 & yes \\
\hline $0059-2735 \ldots \ldots$ & 1.594 & 10848 & & $1246-0542 \ldots \ldots$ & 2.222 & 3790 & \\
\hline $0105-2634 \ldots \ldots$ & 3.488 & 8251 & & $1303+3048^{\mathrm{a}} \ldots \ldots$ & 1.760 & 1682 & yes \\
\hline $0113-4040 \ldots \ldots$ & 2.166 & 416 & & $1309-0536 \ldots \ldots$ & 2.212 & 5773 & \\
\hline $0128-3647 \ldots \ldots$ & 2.169 & 4560 & & $1314+0116 \ldots \ldots$ & 2.690 & 2915 & yes \\
\hline $0134-3740 \ldots \ldots$ & 2.491 & 10877 & & $1331-0108 \ldots \ldots$ & 1.867 & 7400 & \\
\hline $0135-4001 \ldots \ldots$ & 1.853 & 3577 & & $1333+2840 \ldots \ldots$ & 1.907 & 2466 & \\
\hline $0137-0153 \ldots \ldots$ & 2.232 & 3906 & & $1336+1335 \ldots \ldots$ & 2.439 & 5973 & yes \\
\hline $0145+0416 \ldots \ldots$ & 2.029 & 4941 & & $1408+5642 \ldots \ldots$ & 2.562 & 4245 & \\
\hline $0146+0142 \ldots \ldots$ & 2.887 & 5418 & & $1413+1143 \ldots \ldots$ & 2.542 & 6114 & \\
\hline $0226-1024 \ldots \ldots \ldots$ & 2.256 & 7401 & yes & $1414+0859 \ldots \ldots$ & 2.687 & 3516 & \\
\hline $0254-3327 \ldots \ldots$ & 1.862 & 694 & & $1423+5001 \ldots \ldots$ & 2.230 & 3050 & \\
\hline $0321-3344 \ldots \ldots$ & 1.985 & 3305 & & $1442-0011 \ldots \ldots$ & 2.215 & 5314 & \\
\hline $0324-4047 \ldots \ldots$ & 3.058 & 3614 & & $1443+0141 \ldots \ldots$ & 2.446 & 6951 & \\
\hline $0333-3801 \ldots \ldots$ & 2.210 & 3432 & & $1504+1041 \ldots \ldots$ & 3.091 & 4140 & \\
\hline $0335-3339 \ldots \ldots$ & 2.258 & 7515 & & $1524+5147 \ldots \ldots$ & 2.873 & 1449 & \\
\hline $0340-4505 \ldots \ldots$ & 2.004 & 402 & & $1641+4115 \ldots \ldots$ & 2.005 & 1482 & \\
\hline $0828+1229 \ldots \ldots$ & 2.778 & 2507 & yes & $2154-2005 \ldots \ldots$ & 2.028 & 864 & \\
\hline $0842+3431 \ldots \ldots$ & 2.131 & 3650 & & $2201-1834 \ldots \ldots$ & 1.817 & 2049 & \\
\hline $0856+1714 \ldots \ldots$ & 2.323 & 8590 & & $2202-2007 \ldots \ldots$ & 2.188 & 7724 & \\
\hline $0903+1734 \ldots \ldots$ & 2.776 & 9118 & yes & $2212-1759 \ldots \ldots$ & 2.222 & 2221 & \\
\hline $0932+5006 \ldots \ldots$ & 1.914 & 6707 & & $2225-0534 \ldots \ldots$ & 1.981 & 8112 & \\
\hline $1009+0222 \ldots \ldots$ & 1.343 & 1565 & yes & $2240-3702 \ldots \ldots$ & 1.835 & 8539 & \\
\hline $1011+0906 \ldots \ldots$ & 2.262 & 6205 & & $2350-0045 \ldots \ldots$ & 1.626 & 6600 & \\
\hline $1029-0125 \ldots \ldots$ & 2.038 & 1674 & & $2358+0216 \ldots \ldots$ & 1.854 & 6283 & \\
\hline
\end{tabular}

${ }^{\text {a }}$ Coordinate name mislabeled in Table 1 of Weymann et al. 1991, and not reported as such in their Note Added in Proof.

ous results. Two spectral regions were found to be generally robust in the cross-correlation: $1552-2200$ and $2700-2850 \AA$. The first spans the region from the red core of the $\mathrm{C}$ IV emission line, through the emission of $\mathrm{He}$ II $\lambda 1640 / \mathrm{O}$ III] $\lambda 1663$, through the emission of $\mathrm{Al}$ III $\lambda 1858 / \mathrm{C}$ III] $\lambda 1909$, and finally through the generally substantial emission, possibly due to Fe II, near $2080 \AA$. The second spans the broad emission line of Mg II $\lambda 2798$. Areas of broad absorption were avoided for obvious reasons. This meant avoiding certain regions in those few BALQSOs with strong Al III and/or Mg II BALs. The first cross-correlation region has the advantage of covering a large number of pixels, with several weak emission features as well as the strong one near C III] $\lambda 1909$. However, it had the disadvantage that the cross-correlation tended to be dominated by the emission near C III] $\lambda 1909$. This became a problem in those individual objects which have strong emission due to $\mathrm{Al}$ III $\lambda 1858, \mathrm{Si}$ III] $\lambda 1898$ and/or the several transitions due to $\mathrm{Fe} \mathrm{III,}$ which are not strong in the mean spectrum template. The second cross-correlation region had the advantage of being centered upon a generally strong, high contrast, emission line; however, the disadvantage was that it covered a much smaller number of pixels and generally lay in the observed-frame telluric water vapor bands which are difficult to correct for and which often distort the $\mathrm{Mg}$ II emission line profile. Generally, the cross-correlation results from each region matched well. We note that the 13 spectra recently obtained at LCO did not have the spectral coverage to include $\mathrm{Mg}$ II, and in some cases the cross-correlation region between $\mathrm{C}$ IV and $\mathrm{C}$ III] emission covered very few pixels. In two cases (0113-4040 and 03333801 ), the final redshift corrections were in the end determined by eye.

The accuracy with which we could measure the mean broad emission-line redshift varied substantially from object to object. Generally, this internal accuracy was good to within $\sim 400 \mathrm{~km} \mathrm{~s}^{-1}$ or less; we note that the spectral resolution of the Double Spectrograph red camera is $\sim 750 \mathrm{~km} \mathrm{~s}^{-1}$ in the region where a typical C IV BAL trough might fall in many of the spectra. In the worst cases, the internal accuracy was good to only $\sim 1000-1500 \mathrm{~km} \mathrm{~s}^{-1}$. In every case, each BALQSO spectrum was checked by eye against the template after the redshift correction was made, in order to ascertain that the cross-correlation task was not misled by some of the caveats described 

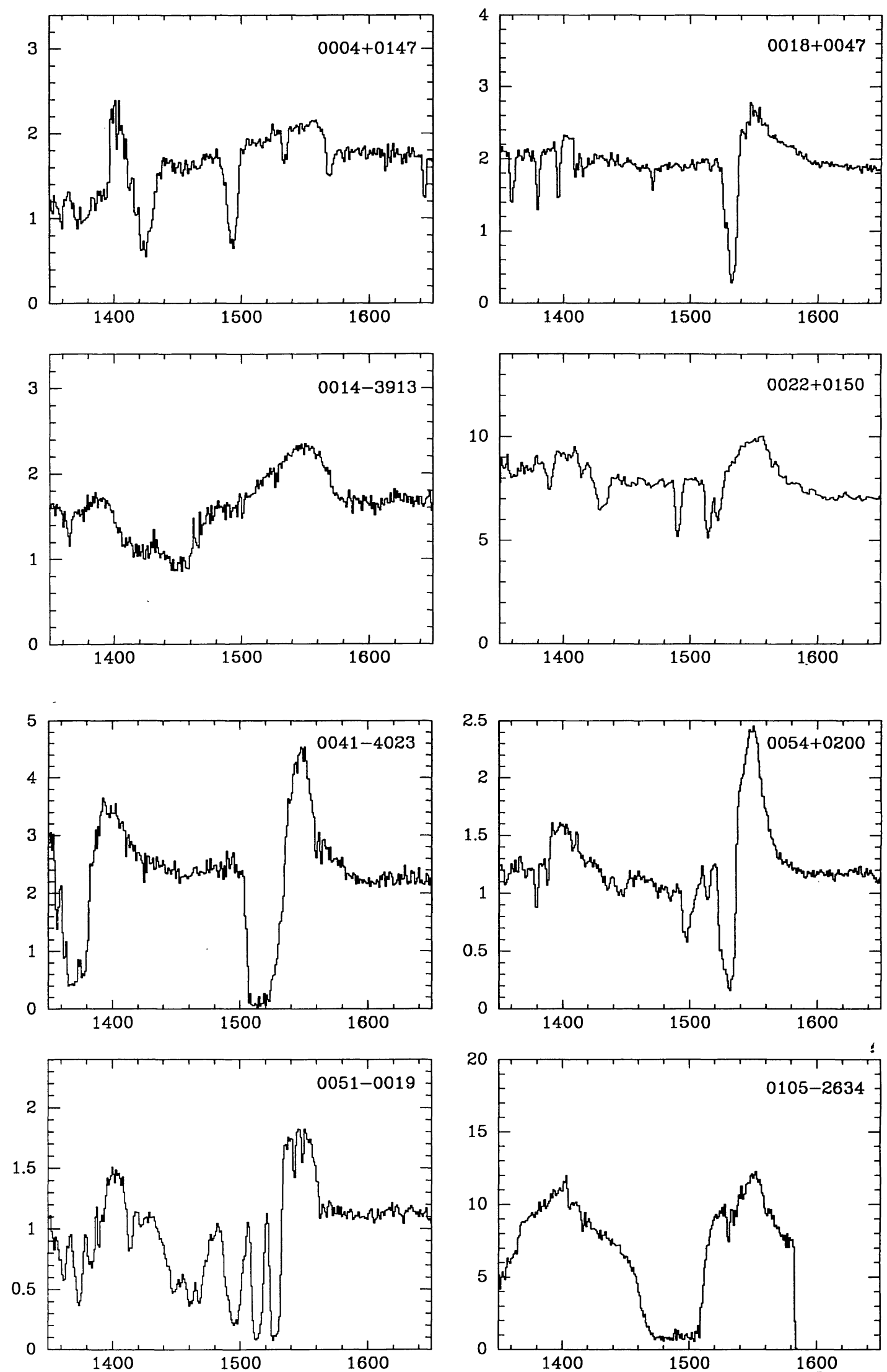

FIG. 1.- (a) Spectra of the 32 new BALQSOs centered on the C IV $\lambda 1549$ BAL, spanning 1350-1650 $\AA$. The data are in the rest frame of the quasar, with the ordinate having units of $10^{43} \mathrm{ergs} \mathrm{s}^{-1} \AA^{-1}$, for $H_{0}=50 \mathrm{~km} \mathrm{~s}^{-1} \mathrm{Mpc}^{-1}$ and $q_{0}=0.5$. Data have been rebinned to $1 \AA$ per pixel (roughly $200 \mathrm{~km} \mathrm{~s}$ at 1500 $\AA$ ). Note that emission due to Si IV $\lambda \lambda 1393.8,1402.8$ and O IV] $\lambda 1402$, and a portion of the broad absorption due to the former ion, appear shortward of $\sim 1400 \AA$ A. (b) Spectra of the 40 BALQSOs in the W91 study. These spectra are presented as in Fig. $1 a$. 

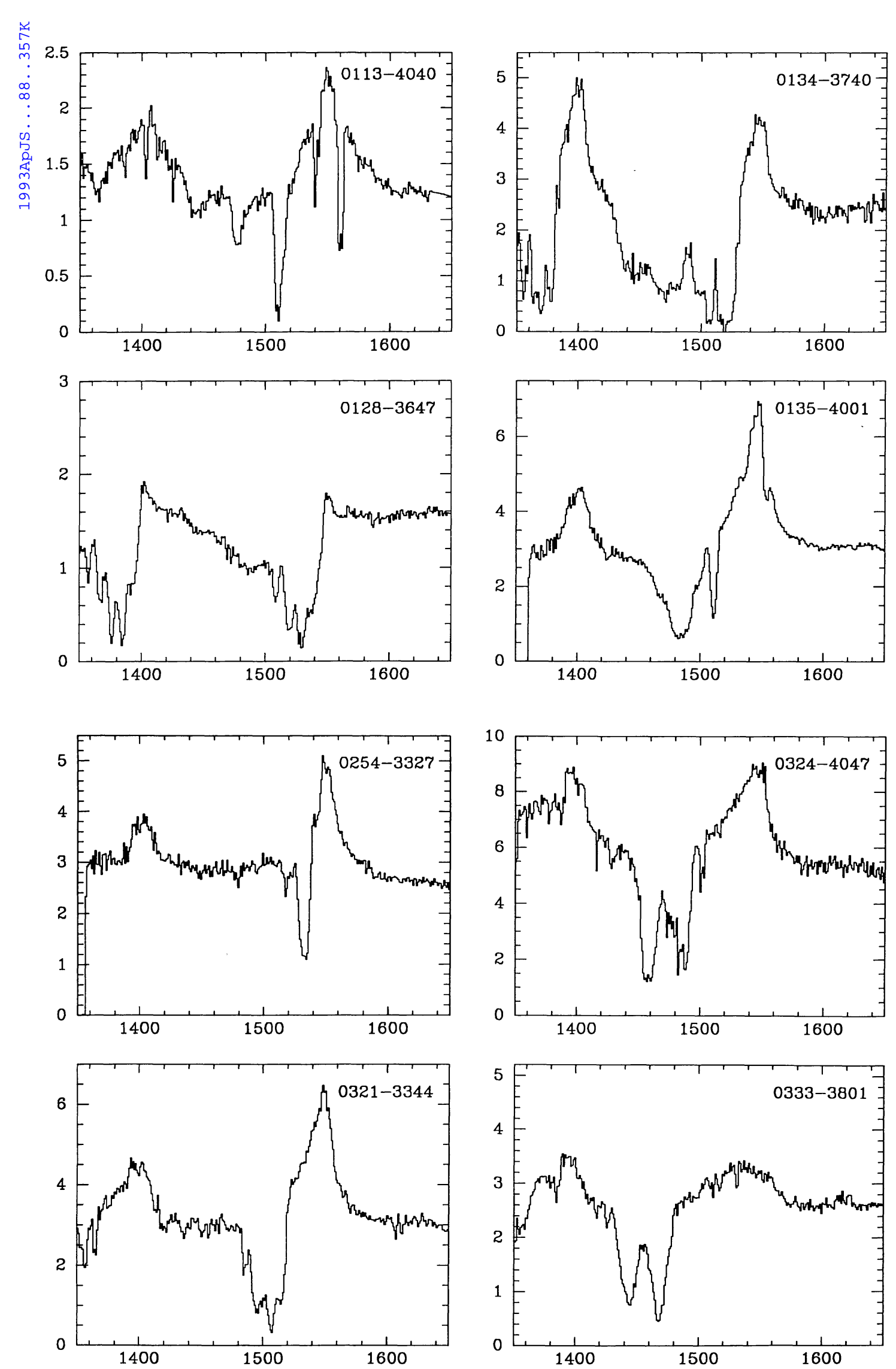

FIG. 1a-Continued 

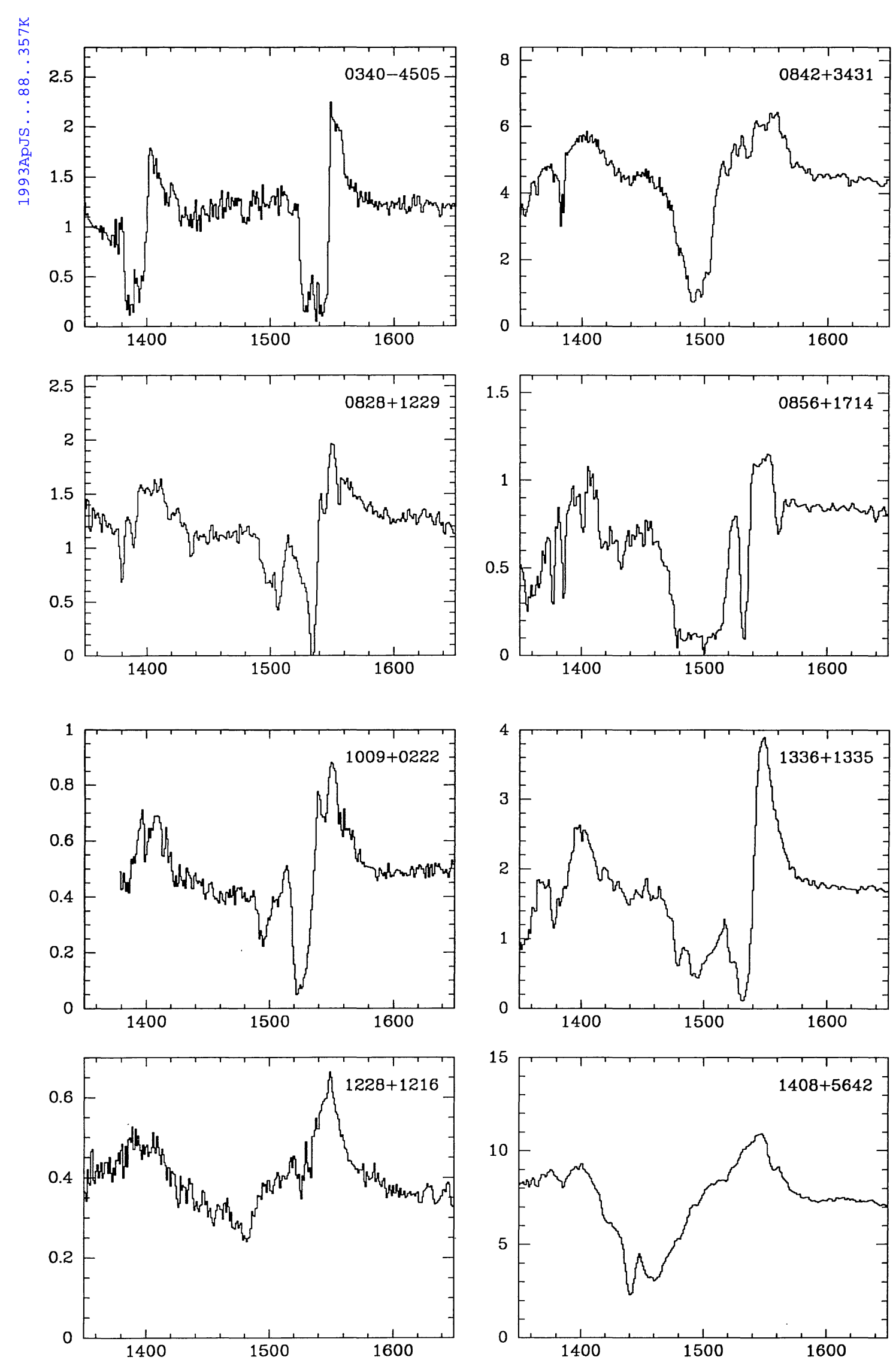

FIG. 1a-Continued 

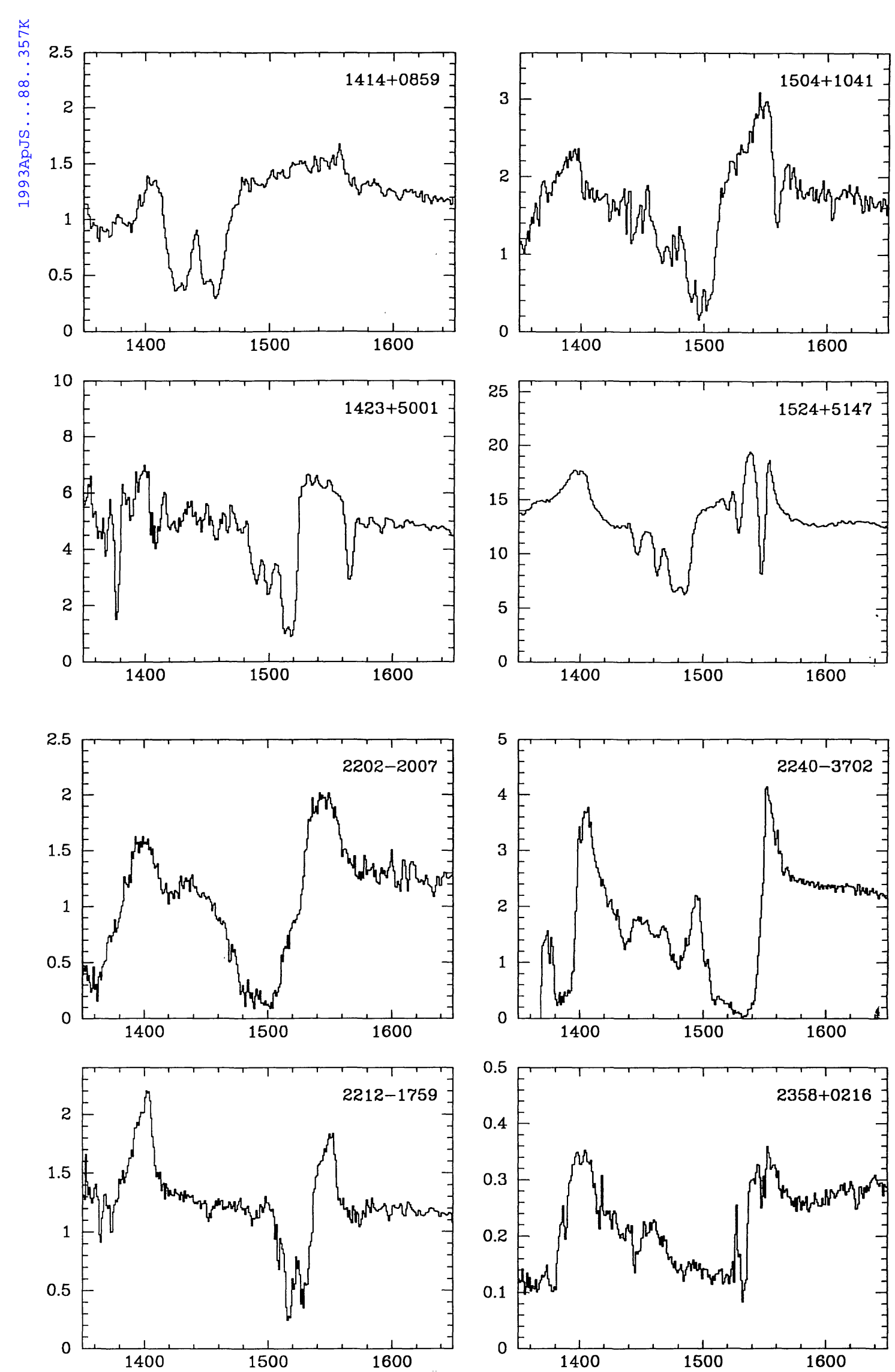

FIG. 1a-Continued 

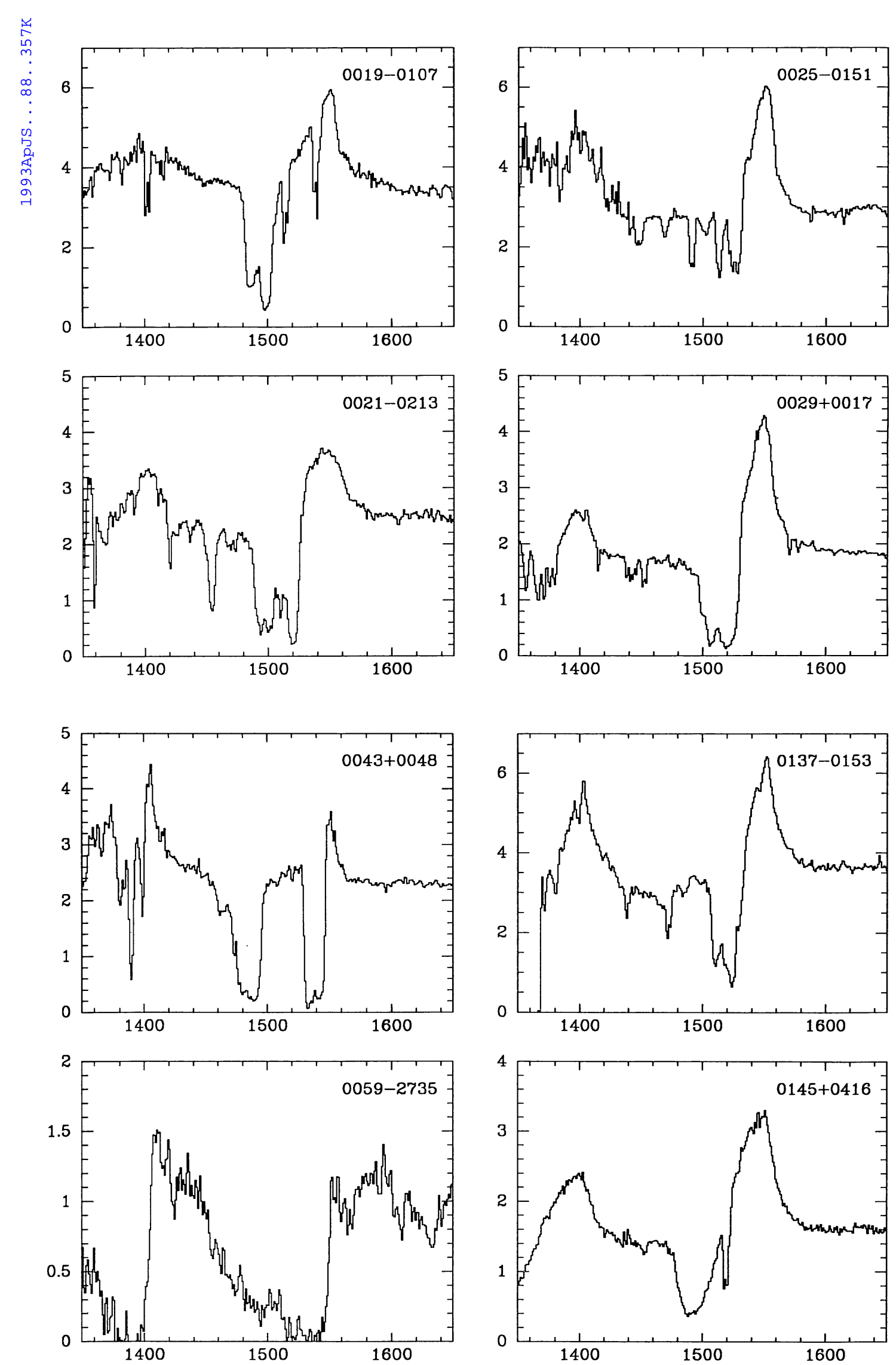

FIG. $1 b$ 

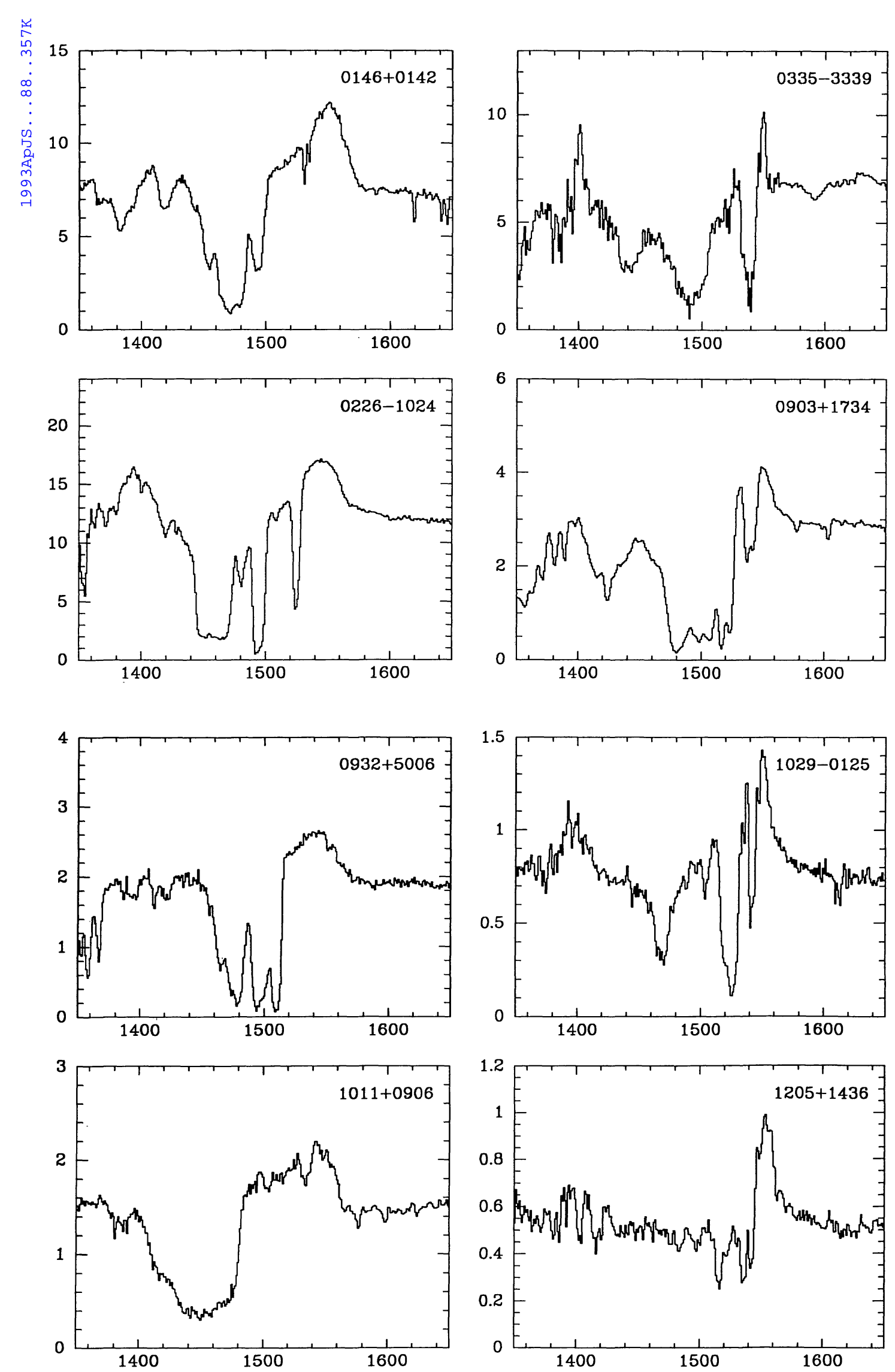

FIG. $1 b-C o n t i n u e d$ 

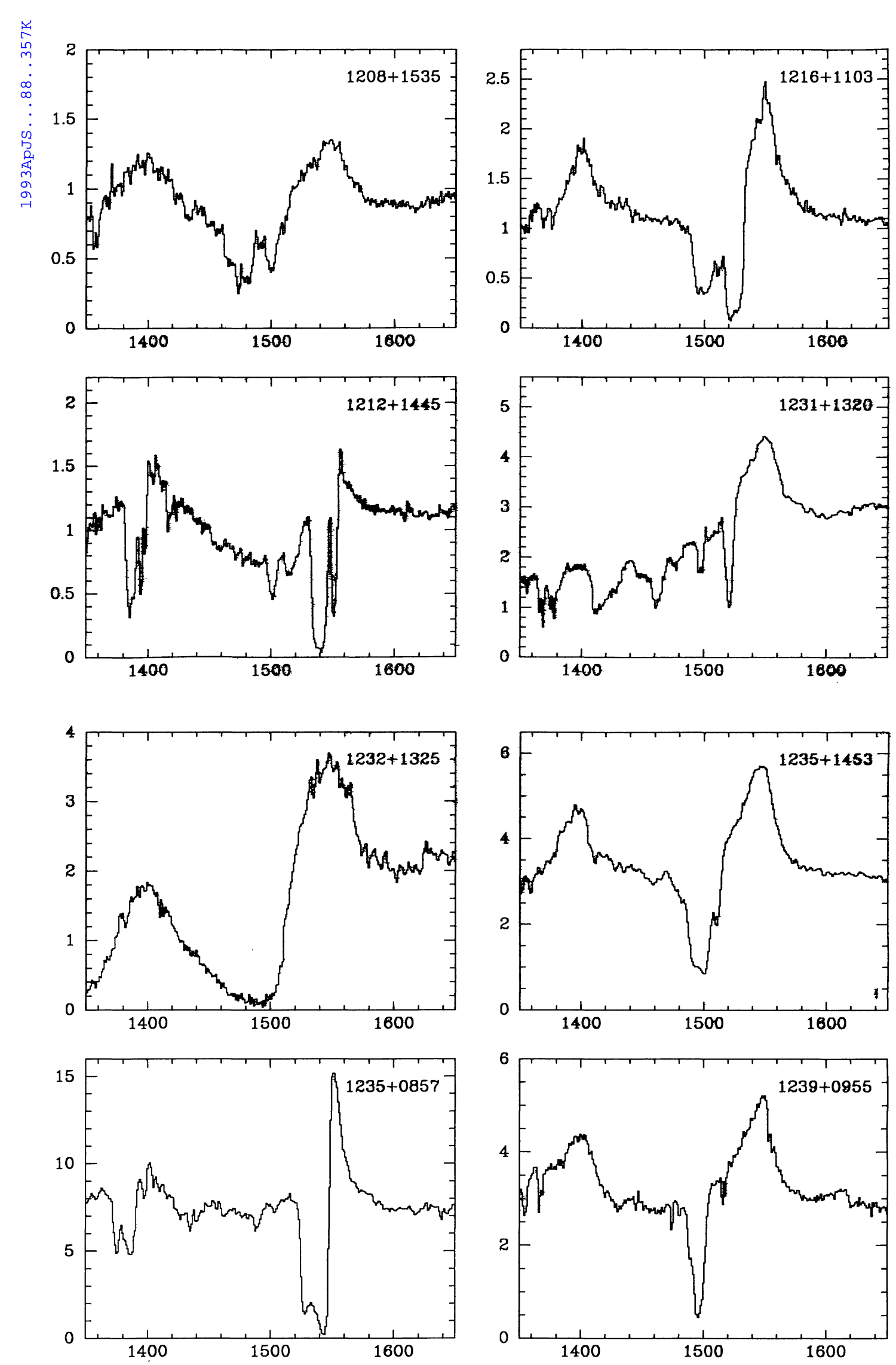

FIG. $1 b$-Continued 

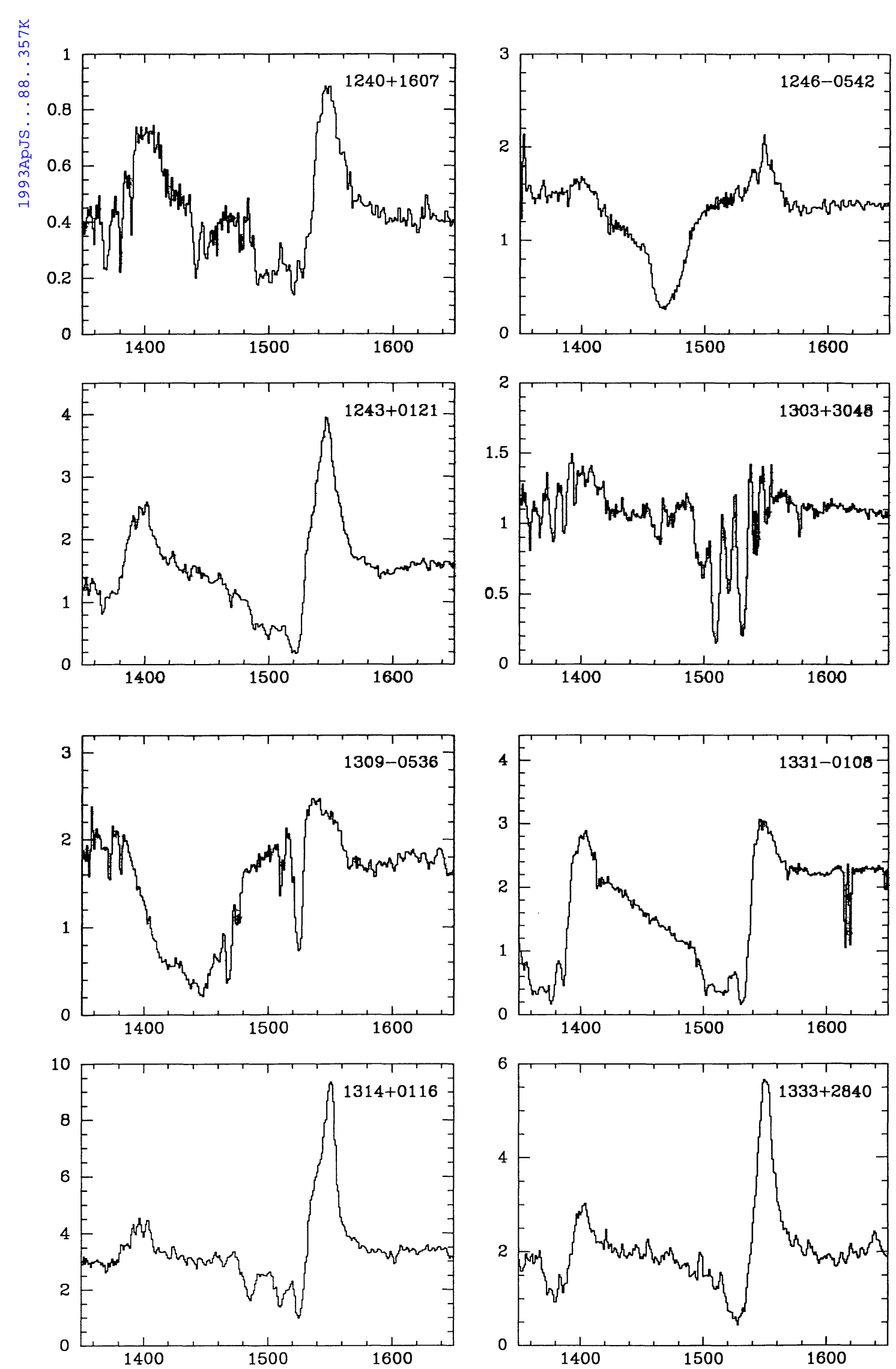

Fig. $1 b$-Continued 

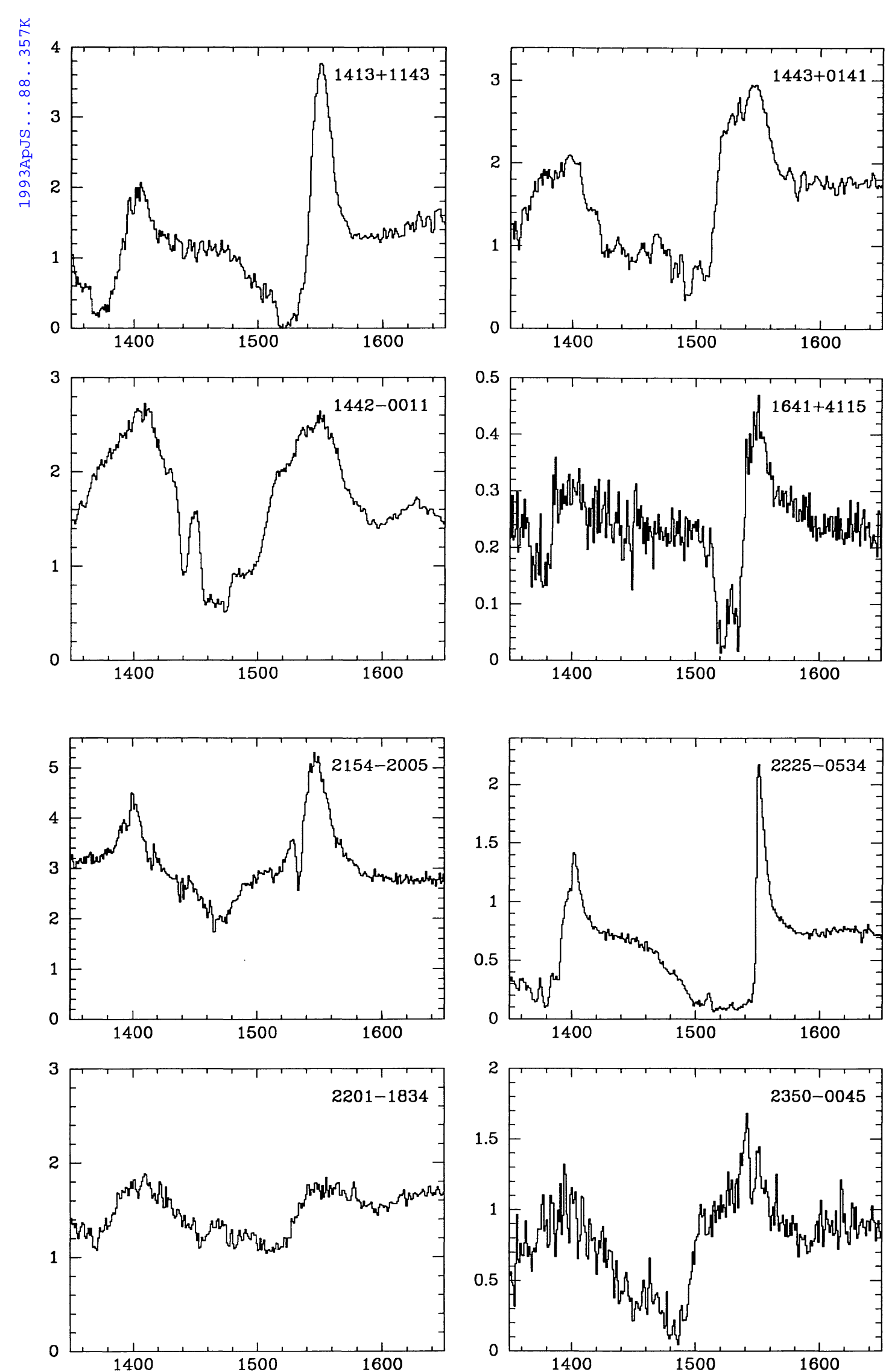

FIG. $1 b-$ Continued 
above. Only 18 of the 72 BALQSOs had redshift corrections larger than $400 \mathrm{~km} \mathrm{~s}^{-1}$ (absolute value). Eight of these 18 redshift corrections were still considered very uncertain (with corrections ranging from $400-2500 \mathrm{~km} \mathrm{~s}^{-1}$ ). Five of these 18 (and three of the eight) were from the recent LCO observations with limited spectral coverage. The mean and rms redshift corrections (absolute) were both $\sim 400 \mathrm{~km} \mathrm{~s}^{-1}$; the median was roughly half that.

A new mean BALQSO spectrum was derived using the updated redshifts, and the process described above was repeated for all spectra using the new mean spectrum as the template, with very little change in the results. The final redshifts obtained by this iterated cross-correlation method are presented in column (2) of Table 1B. We refer to these redshifts as defining a systemic rest frame, but there may well be differences of a few hundred $\mathrm{km} \mathrm{s}^{-1}$ between these redshifts and those which might be measured by forbidden lines.

The final mean spectrum of 72 BALQSOs is shown in Figure 2. We note that only in the region spanning the $\mathrm{C}$ IV BAL trough $(\sim 1400-1550 \AA)$ do all 72 BALQSO spectra contribute. For this reason, and because other troughs are either distinctly weaker (e.g., $\mathrm{Si}$ IV) and/or of poorer $\mathrm{S} / \mathrm{N}$ or badly blended (e.g., $\mathrm{N} \mathrm{v}$ and $\mathrm{Ly} \alpha$ ) we chose to illustrate in Figures $1 a$ and $1 b$ only the region covering the $C$ IV trough. We should emphasize though, that provided there are not quite strong local variations in the ionization level with velocity (and we can think of no very plausible reason why this should be) then the fine-scale velocity structure in the trough of one ion will be present in the troughs of the other ions (cf. Korista et al. 1992). In particular, though we consider in $\S 5$ the evidence for line locking which involves the N v $\lambda 1240 \AA$ and Ly $\alpha$ transitions, the evidence for (or against) this possibility will be exhibited just as strongly in the $\mathrm{C}$ IV trough as in the $\mathrm{N} \mathrm{V}$ and Ly $\alpha$ troughs.

All BALQSOs in this study have nonzero "balnicity indeces", as defined in W91, the lowest being $224 \mathrm{~km} \mathrm{~s}^{-1}$ for $0022+0150$, which is $\sim 8$ times larger than the balnicity index of 2211-1915, listed as a "marginal" BALQSO in W91. The balnicity index (BI), which is a modified velocity equivalent width $\left(\mathrm{km} \mathrm{s}^{-1}\right)$ of the $\mathrm{C}$ IV BAL as measured between -3000 and $-25,000 \mathrm{~km} \mathrm{~s}^{-1}$, and can range from $0 \mathrm{~km} \mathrm{~s}^{-1}$ (no BAL) to a maximum of $20,000 \mathrm{~km} \mathrm{~s}^{-1}$, is given in column (3) of Table 1B for each of the BALQSOs. Note that only contiguous absorption, with residual intensities less than 0.9 , spanning at least $2000 \mathrm{~km} \mathrm{~s}^{-1}$ contributes to the balnicity index.

\section{THE STRUCTURE IN THE MEAN C IV TROUGH: REAL?}

Several types of analyses were utilized in an attempt to test whether or not the structure in the composite C IV BAL in W91 could be due to statistical fluctuations.

\subsection{The Mean Residual Intensity Spectrum}

Since the optical depth parameterizes the column density of ions through the broad absorption-line region, we will present our data in the form of residual intensity $\left[I_{r}(\lambda)=e^{-\tau(\lambda)}\right]$,

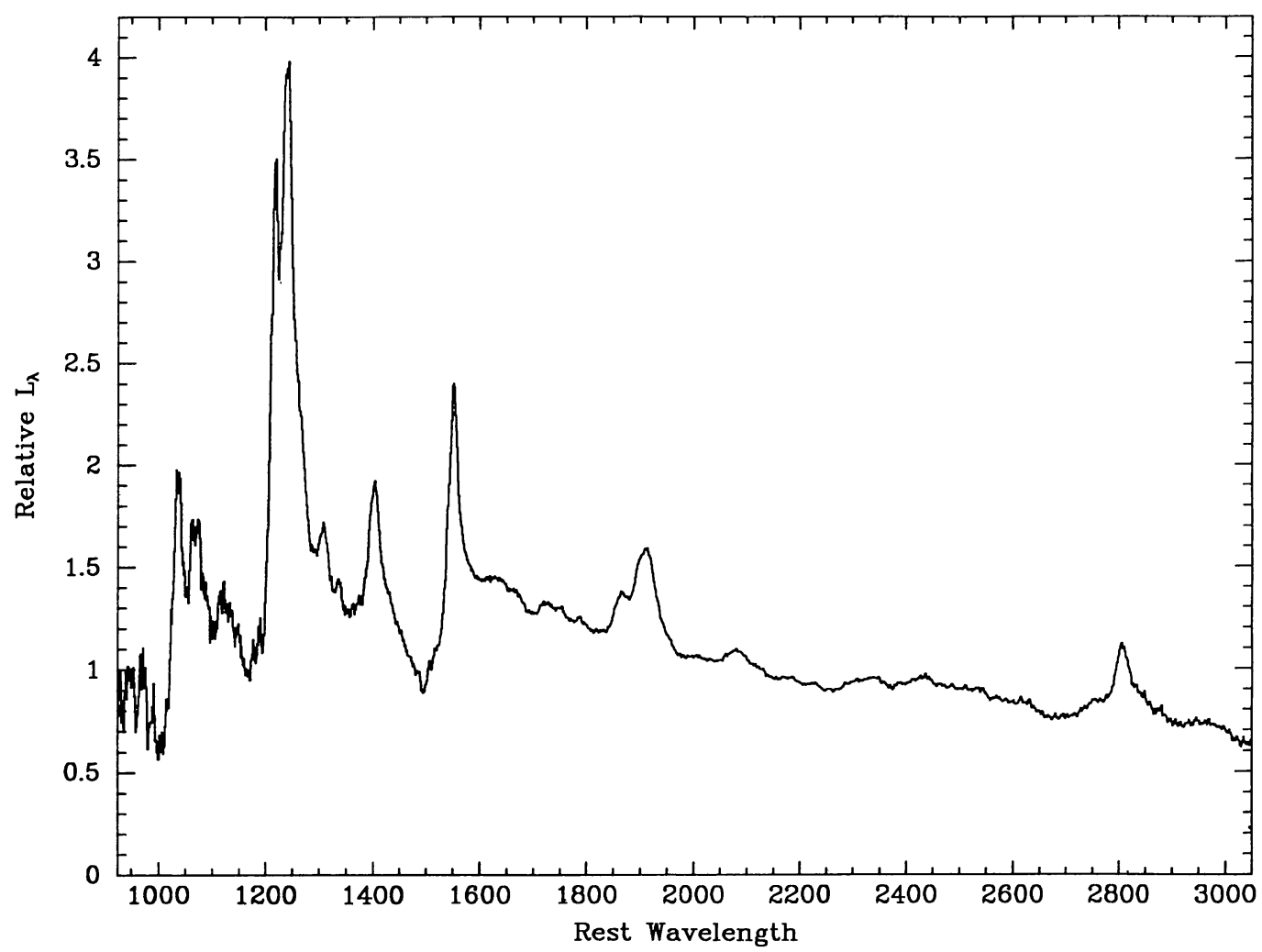

FIG. 2.-Mean BALQSO rest-frame spectrum, composed of all 72 BALQSO spectra, after the corrections to the redshifts have been made. The ordinate has units of relative luminosity (ergs s$\left.{ }^{-1} \AA^{-1}\right)$. Note that only in the region spanning the C IV BAL trough $(\sim 1400-1550 \AA)$ do all 72 BALQSO spectra contribute. 
rather than as a difference in flux (between BALQSO and nonBALQSO mean spectra). That is, we attempted to remove the emission lines and continuum from each BALQSO through division by a non-BALQSO emission-line and continuum template, which we take to represent the effective continuum seen by the BAL clouds. The template used was the high $\mathrm{S} / \mathrm{N}$ mean non-BALQSO spectrum of sample 1' from W91. Korista et al. (1992) describe the assumptions made when deriving the residual intensity in this manner. We note that the resultant residual intensities in subtrough 1 will be more sensitive to uncertainties in the effective continuum than those in subtrough 2, because subtrough 1 lies in the blue wing of the broad emission. In particular, we (qualitatively) place less weight on the residual intensity for outflow velocities less than $3000 \mathrm{~km}$ $\mathrm{s}^{-1}$, since in this region the residual intensity is very sensitive to the match between the template emission and the emission intrinsic to the individual BALQSO. In doing so we also attempt to avoid confusion with the so-called "associated" absorption systems, usually occurring at or very near to $z_{\mathrm{em}}$, which are thought to be unrelated to the BAL phenomenon. After suitably scaling the BALQSO spectrum to the template, the latter was divided into the former, and a low-order polynomial was then fit to BAL-free regions in an attempt to account for differences in continuum shape between the template and the BALQSO.

The mean and median $\mathrm{C}$ IV BAL residual intensity of the total sample of 72 BALQSOs are shown in Figure 3 (we note in passing that this result is virtually identical to the residual in- tensity of the mean and median BALQSO spectra). For comparison, Figure 4 shows the mean and median residual intensity of the sample of 34 BALQSOs shown in the difference spectrum of Figure 5 in W91 (after the redshift corrections); we refer to this as the W91 sample. Comparing Figures 3 and 4 one notes immediately that although the same structure is present, the contrast of subtroughs 1 and 2 relative to the largescale broad absorption envelope (the overall smooth trend from -3000 to $-30,000 \mathrm{~km} \mathrm{~s}^{-1}$ ) has diminished after doubling the sample size; subtrough 1 is much less distinct in the total sample. The reason for this change is demonstrated in Figure 5 where we show the mean and median residual intensity spectra of the 38 new BALQSO spectra only, including the six low-ionization BALQSO spectra presented in W91 but not used in their Figure 5. Structure is present near subtroughs 1 and 2, but both subtroughs are more smeared out in outflow velocity and the strong low-velocity feature is shifted from the position of subtrough 1 in Figure 4. Structure at other velocities is also present, including a much stronger example of a subtrough at intermediate velocities $\left(\sim-7600 \mathrm{~km} \mathrm{~s}^{-1}\right)$ in the W91 sample in Figure 4. These simple comparisons would seem to indicate that a substantial fraction of the total sample of BALQSOs do not exhibit the double troughs; indeed this is borne out by visual inspection of the individual BALQSO spectra.

\subsection{Two Randomly Chosen Subsamples}

We first tested the reality of the double troughs by dividing the total BALQSO sample of 72 into two equal subsamples

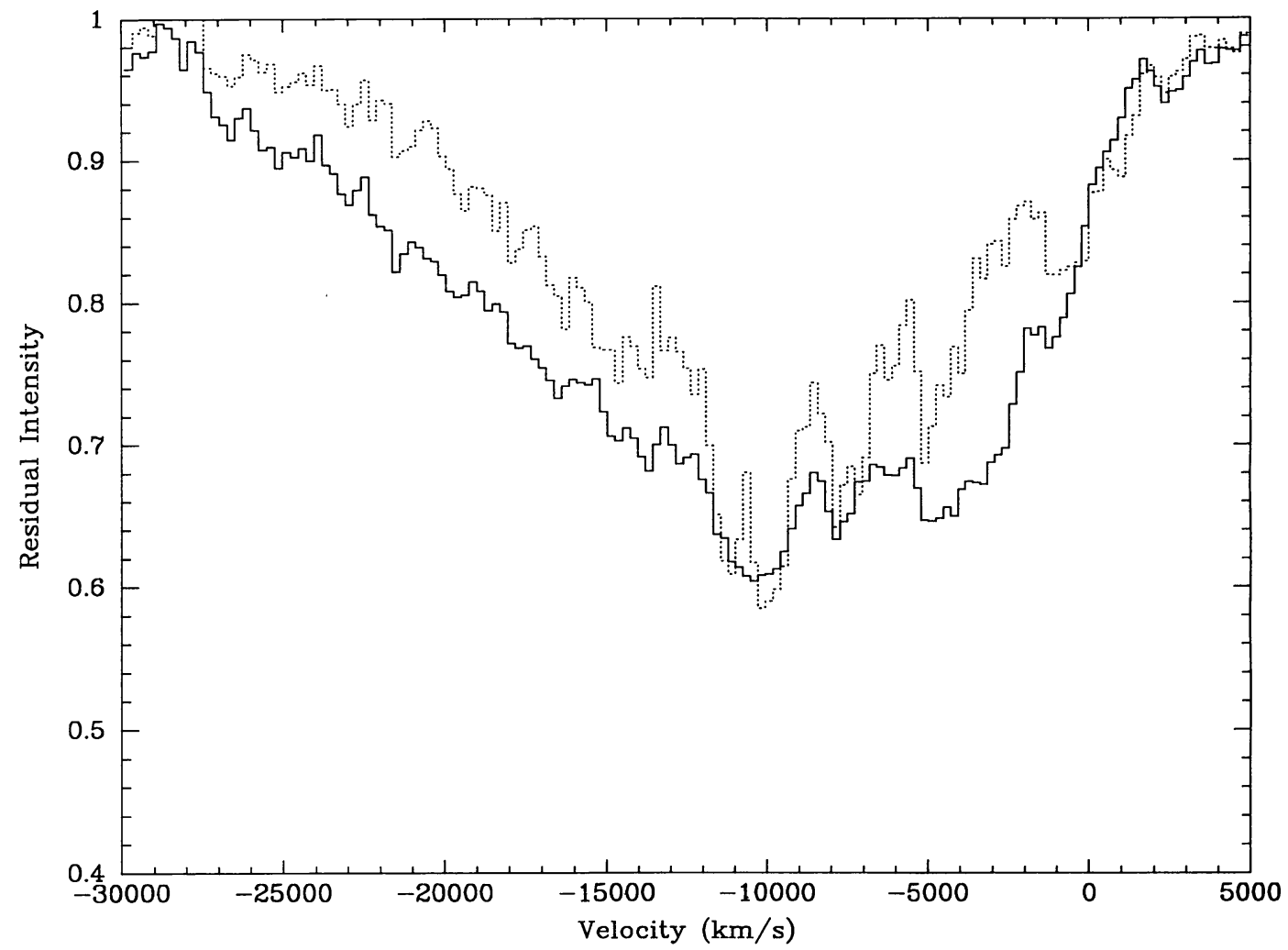

FIG. 3.-Mean (solid line) and median (dotted line) C IV BAL residual intensity spectra for the total sample of 72 BALQSOs. The abscissa has units of radial velocity in $\mathrm{km} \mathrm{s}^{-1}$, relative to the stronger transition of the $C$ IV doublet $\lambda 1548.2$. This will be the case for all figures using a velocity scale. Compare to Fig. 4. 


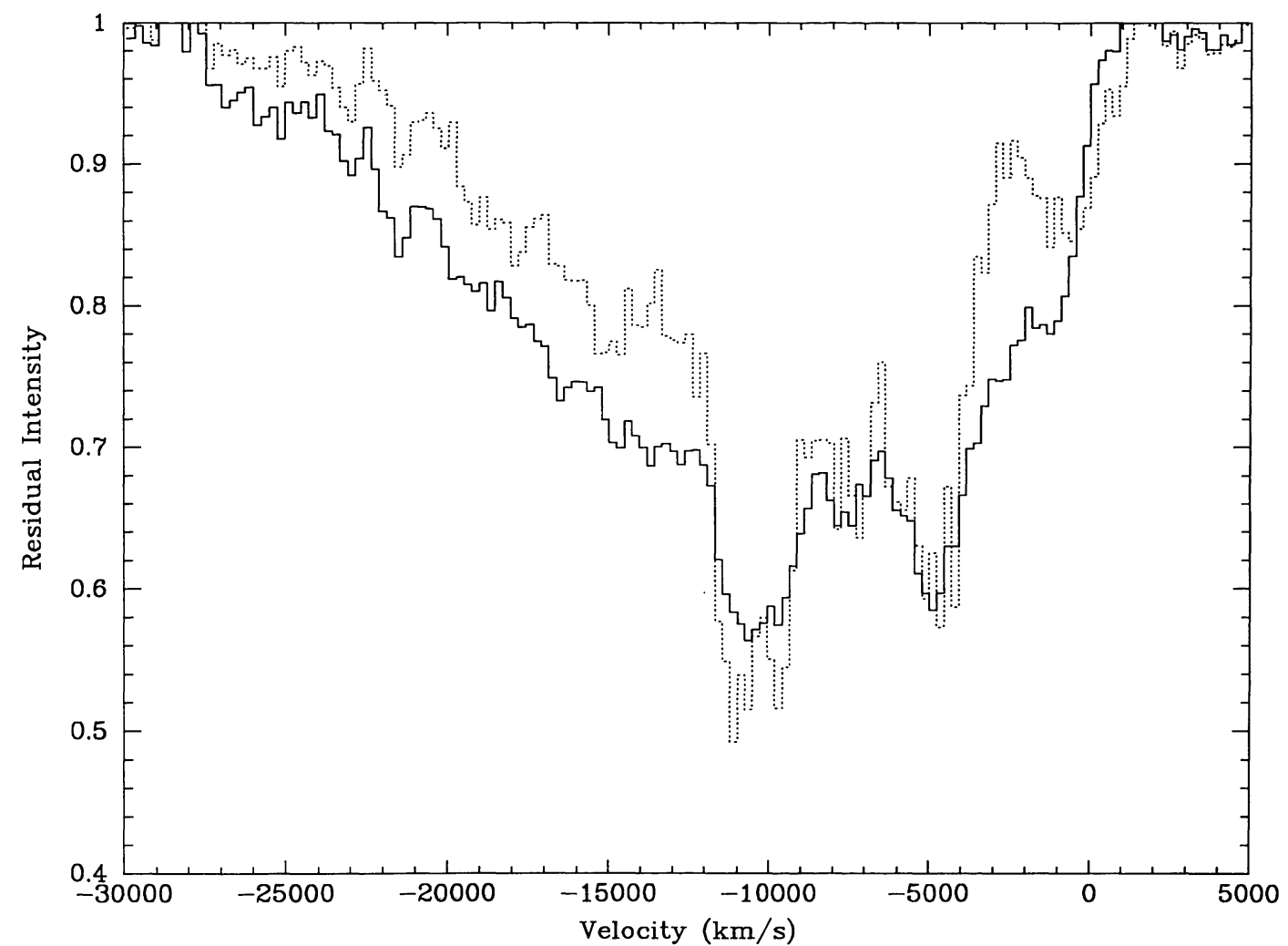

FIG. 4.-Mean (solid line) and median (dotted line) C IV BAL residual intensity spectra for the W91 sample of 34 BALQSOs. Note the two prominent subtroughs near $-10,500 \mathrm{~km} \mathrm{~s}^{-1}$ and $-4700 \mathrm{~km} \mathrm{~s}^{-1}$, as observed by W91 in their difference spectrum.

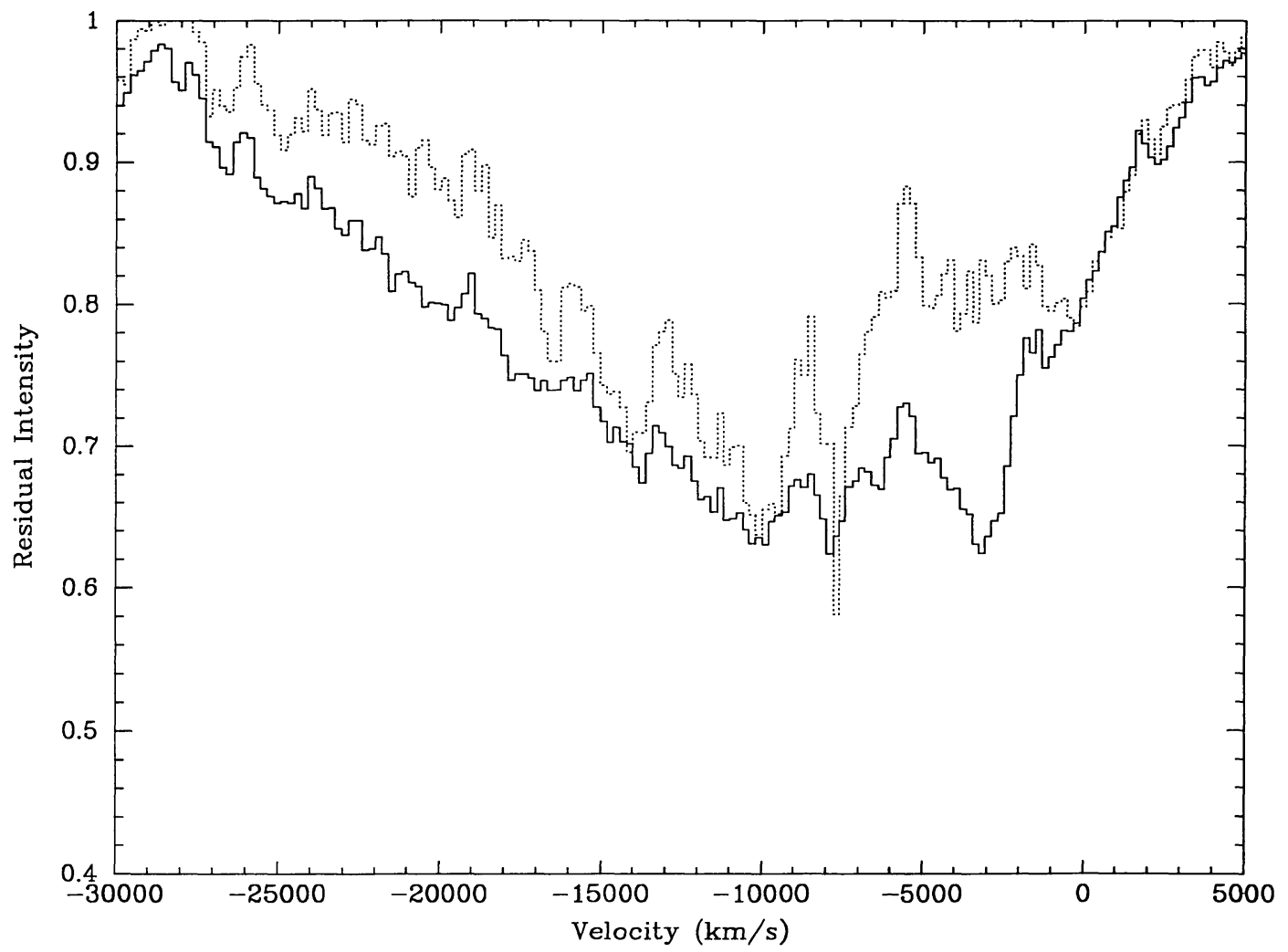

FIG. 5.-Mean ( solid line) and median (dotted line) C IV BAL residual intensity spectra for the 38 BALQSOs in the new sample. This includes the 32 new BALQSO spectra and the six Mg II BALQSOs from the W91 study, but not included in their Fig. 5. Compare to Fig. 4. 

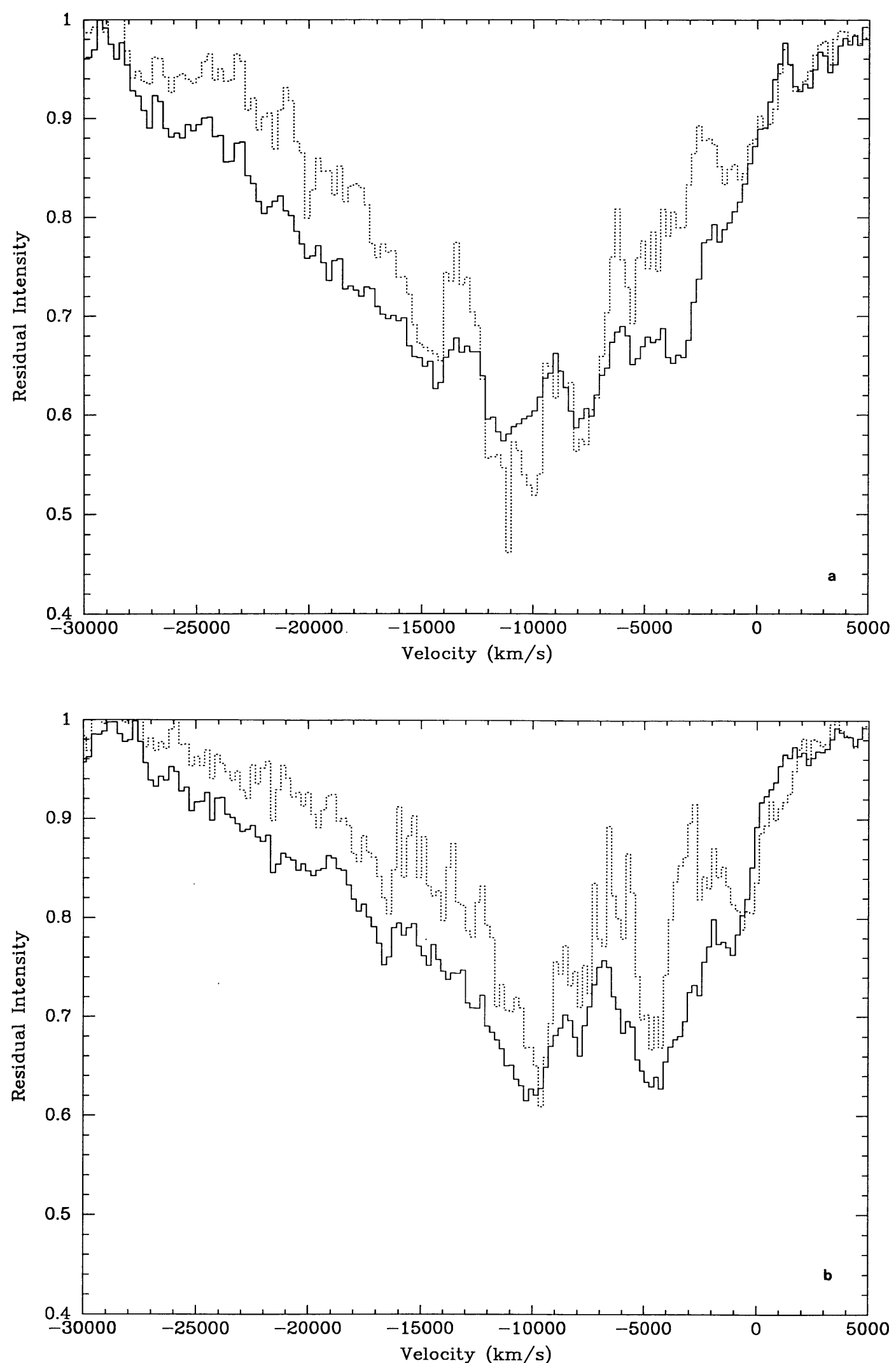

FIG. 6.- Mean (solid line) and median (dotted line) C IV BAL residual intensity spectra for subsamples $(a) 1$ and $(b) 2$, each composed of 36 BALQSOs chosen from the total sample as described in the text. 
whose members were chosen at random from the total sample. This was accomplished by ordering the BALQSOs in right ascension and placing even numbered objects into one subsample and odd into another so that all observing runs at all telescopes would be fairly equally represented in each subsample. The mean and median residual intensity spectra for the two randomly chosen subsamples, labeled subsamples 1 and 2, are shown in Figures $6 a$ and $6 b$. Both subsamples show the double-trough structure, and yet show significant differences from each other as well. For example, subsample 1 (Fig. $6 a$ ) also shows structure at intermediate and high velocities in addition to subtroughs 1 and 2. Also, subtrough 1 is more smeared out in outflow velocity. Subsample 2 (Fig. $6 b$ ) exhibits subtroughs 1 and 2 much more strongly, with some evidence for the presence of the intermediate velocity subtrough. The differences in the details of the mean residual intensities between the two subsamples is an indication that each is influenced by statistical fluctuations expected within a small sample.

\subsection{A Few More Subsamples}

For illustrative and quantitative purposes, we have constructed two other subsamples. In Figure 7 we show the result of randomly perturbing the redshifts of the 72 individual BALQSO spectra over $\pm 3 \sigma$, where $\sigma$ is roughly the half-width of a subtrough (i.e., $1250 \mathrm{~km} \mathrm{~s}^{-1}$ ). This procedure is intended to remove subtrough velocity coherence, so as to produce an average spectrum constructed from $72 \mathrm{C}$ IV BAL profiles with incoherent structure. Note that the mean is not perfectly smooth, although the contrast in any feature from the largescale BAL envelope is certainly much smaller than in any of the subsamples formed thus far. We will refer to this as the "random- $z$ " sample.

In Figure $8 a$ we show the resultant mean and median residual intensity spectra of 16 BALQSOs, as indicated in column (4) of Table 1B, which were chosen by eye to have a pair of distinct troughs within $\pm 1000 \mathrm{~km} \mathrm{~s}^{-1}$ of 4300 and $10,300 \mathrm{~km}$ $\mathrm{s}^{-1}$, respectively, in outflow velocity. In other words, we have chosen objects having BAL troughs similar to that shown in Figure 4. A "distinct" trough is defined to have these attributes: (1) it must have a residual intensity $\leq 0.8$, the limit chosen to be the mean C IV BAL residual intensity (averaged over outflow velocities between 0 and $30000 \mathrm{~km} \mathrm{~s}^{-1}$ ) in Figure 4; (2) it must have a FWHM $1000-3000 \mathrm{~km} \mathrm{~s}^{-1}$, as measured by the two bordering local maxima, which rise at least 0.1 residual intensity units higher than the local minimum; (3) a wider trough may be counted as having two or more distinct troughs if within this trough the residual intensity recovers by 0.1 or more, like the recovery between the double troughs in Figure 4 . These objects generally have broad absorption at other outflow velocities as well, and only 11 out of the 16 have double troughs with velocity splittings between 5600 and $6500 \mathrm{~km} \mathrm{~s}^{-1}$ (the separation between Ly $\alpha$ and the $\mathrm{N} v$ doublet), as measured by their minima. The remainder have splittings from $\sim 5000-5600$ and $\sim 6500-7000 \mathrm{~km} \mathrm{~s}^{-1}$, although other parts

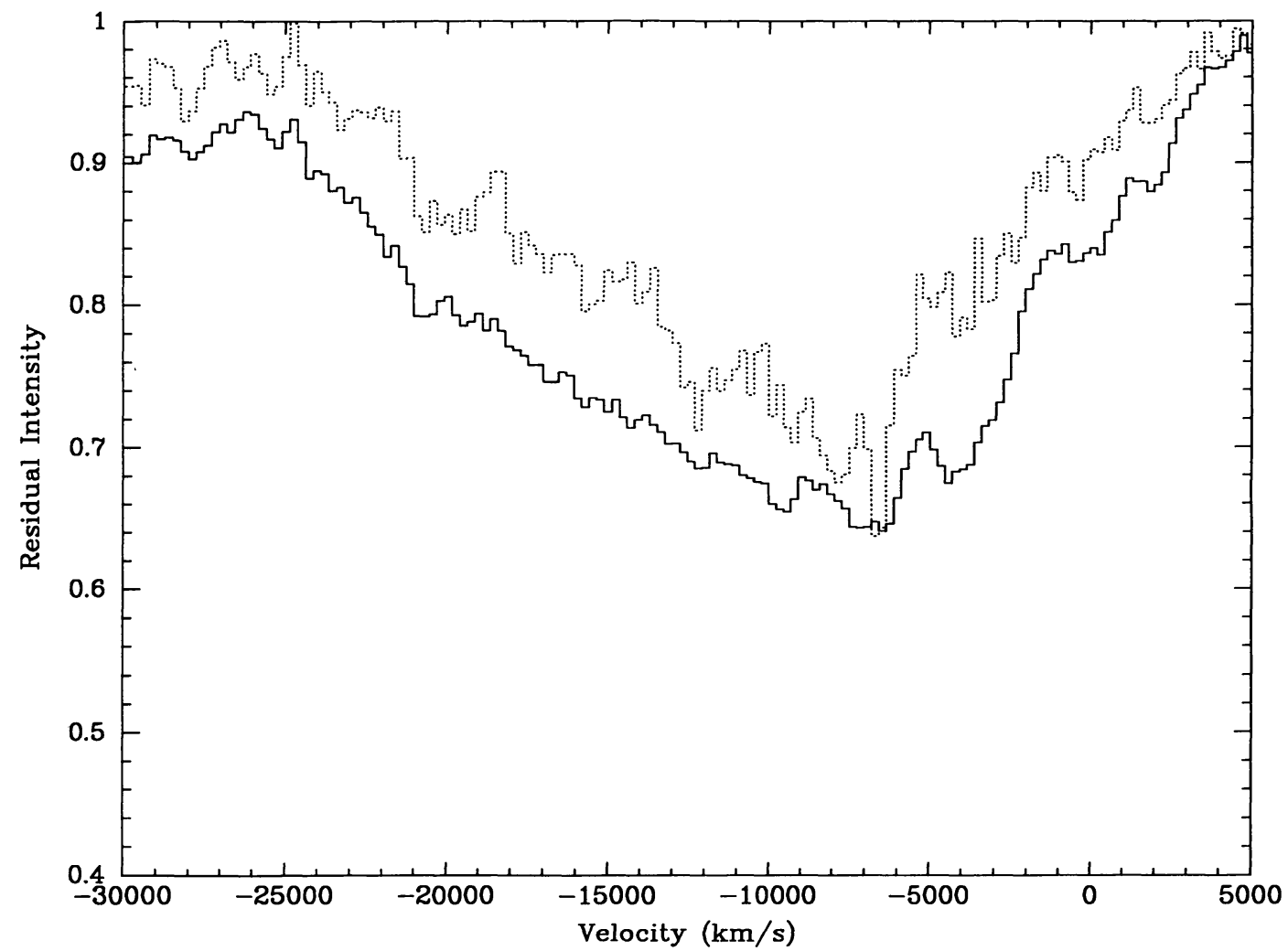

FIG. 7.-Mean ( solid line) and median (dotted line) C IV BAL residual intensity spectra for the "random-z" sample, derived by uniformly randomizing the redshifts of the BALQSOs over $\pm 3 \sigma$, where $\sigma$ is $1250 \mathrm{~km} \mathrm{~s}^{-1}$, roughly equal to one-half the width of the subtroughs in Fig. 4 . Note the general lack of structure within the BAL trough. 

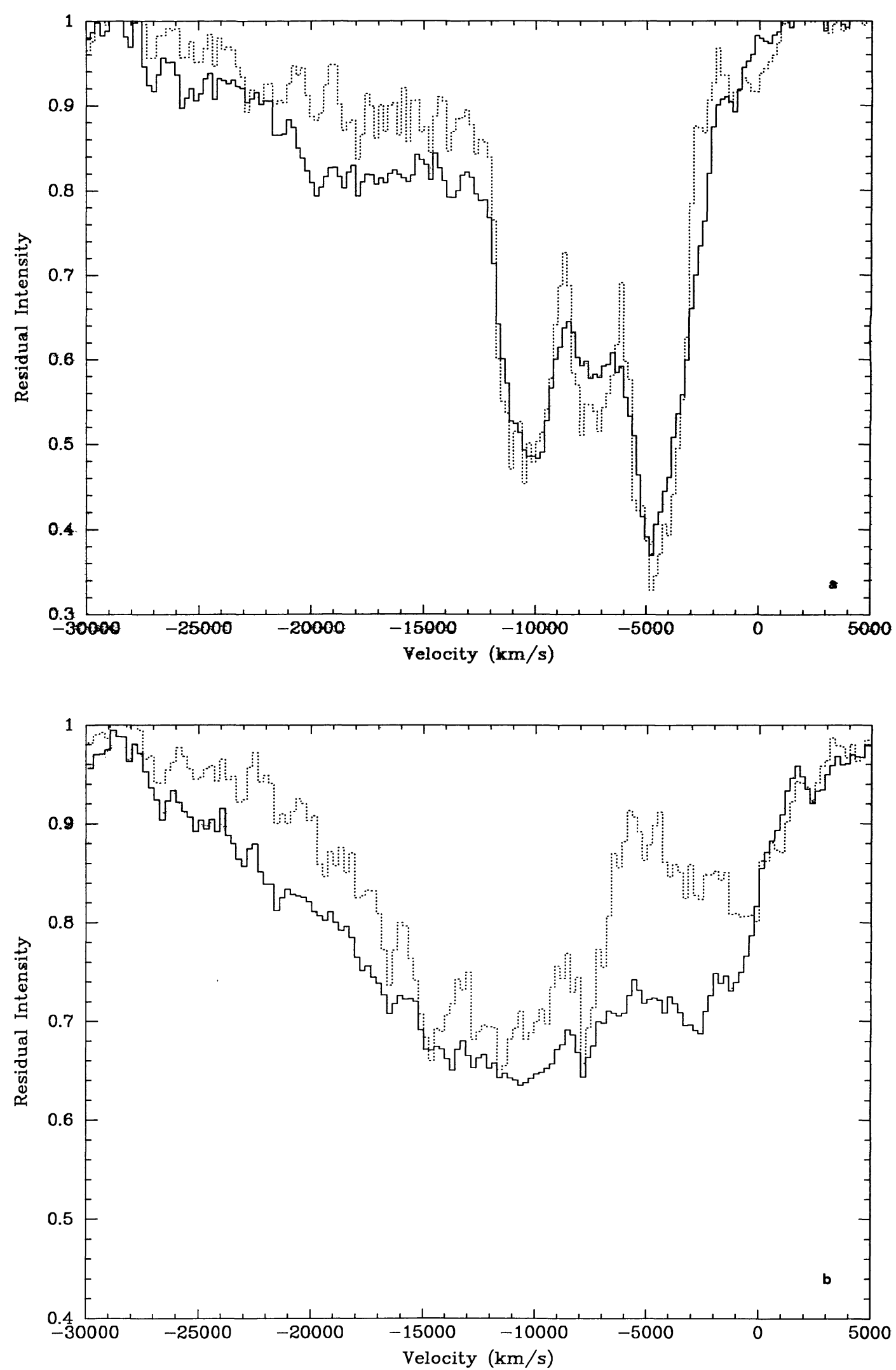

FIG. 8.- (a) Mean ( solid line) and median (dotted line) C Iv BAL residual intensity spectra for the "double-trough" sample of 16 BALQSOs, selected to exhibit explicitly the double trough structure within the broad absorption line as seen in Fig. 4. (b) Mean (solid line) and median (dotted line) C rv BAL residual intensity spectra for the remaining 56 BALQSOs which do not explicitly exhibit double-troughed BALs. Note the general lack of structure. 
of these troughs did have the Ly $\alpha-\mathrm{N} v$ splitting. We emphasize that tests of the reality of the double troughs will not be conducted on this manifestly biased sample.

As expected, the "signal" in subtroughs 1 and 2 is very much higher than in any of the subsamples formed thus far. The intermediate velocity subtrough is also apparent. We will refer to this as the "double-trough" sample. It represents $22 \%$ of the total BALQSO sample and is responsible for most of the double-trough structure observed in the total sample. Indeed, we can see little evidence of the type of structure seen in Figures 3 , 4 , and $8 a$ in either the mean or median spectrum of a sample constructed from the remaining 56 BALQSOs, shown in Figure $8 b$. Finally, we note that there are no BALQSOs within the total sample with optical depths greater than 2 over the span of the two subtroughs, and there are only three objects with optical depths greater than 2 over half that span in outflow velocity. Thus it is unlikely that this double-trough phenomenon occurs unnoticed in objects with very deep troughs.

One should not infer from the smoothness of the BAL profile in Figure $8 b$ that this "non-double-trough" sample is composed of BALQSOs whose BALs are structureless (e.g., one can see evidence of the aforementioned intermediate velocity trough near $-7600 \mathrm{~km} \mathrm{~s}^{-1}$ ). On the contrary, roughly twothirds of the BALQSOs in this sample show structure having the three characteristics defining a "discrete" trough described just above, but they occur at different outflow velocities. In fact a few of these BALQSOs have discrete double troughs, as defined for the "double-trough" sample, which have a velocity splitting roughly that of $\mathrm{Ly} \alpha-\mathrm{N} v$, but they occur at outflow velocities different from those which appear in Figure 4 (e.g., in 0113-4040, 0324-4047, 1414+0859). Parenthetically, we note that in three cases double troughs occur in addition to those which appear in Figure 4 (e.g., 0051-0059, 0226-1024, $1303+3048$ ).

Section 5 will discuss how BAL trough pairs might originate through line-locking. As just described, it is conceivable that pairs of troughs with similar velocity separations could occur at velocities other than the pair which appear in Figure 4. To test all double-trough pairs for line locking relative to the initial (lowest velocity) BAL trough, the redshifts of all spectra were modified such that the red edge of the first BAL trough of each BALQSO spectrum would lie coincident with that of every other. The "red edge of the first BAL trough" is defined to be where the residual intensity first falls below 0.75 and remains below this level over more than $1000 \mathrm{~km} \mathrm{~s}^{-1}$. With these qualifications, we hope to exclude non-BAL absorption. Only data having outflow velocities less than $30,000 \mathrm{~km} \mathrm{~s}^{-1}$ in the quasar rest-frame are included in order to avoid contamination from the $\mathrm{Si}$ IV trough and residual $\mathrm{Si}$ IV emission. The resulting mean and median residual intensity spectra of the total BALQSO sample are shown in Figure $9 a$. The mean spectrum is nearly featureless; if line-locking were linked to the velocity of the initial trough, one would expect a second minimum near $-7000 \mathrm{~km} \mathrm{~s}^{-1}$ in Figure $9 a$.

The distribution of the velocities of the first trough (Fig. $9 b$ ) shows no preferred velocities, which demonstrates that the double-trough structure does not result from some objects having a single trough at the position of one of the subtroughs and others having a single trough at the position of the other subtrough (i.e., a bimodal distribution of subtroughs). The above procedure was also applied to the strongest trough, and the results were nearly identical (in many cases the first trough, as defined above, is the strongest trough ). The implication of this experiment is that whatever the mechanism responsible for the double troughs, it must, as a general rule, be tied to some absolute reference frame in the quasar, rather than to the frame of any particular BAL trough.

As an aside comment, the shape of the trough in Figure $9 a$ is interesting in itself. It clearly demonstrates that once a BAL sets in, it quickly deepens, and more slowly decays at higher velocities. It defines a smooth BAL optical depth envelope common to BALQSOs, upon which the structural details of absorption within individual BALQSOs are superposed.

\subsection{More Quantitative Measurements}

The investigations of the reality of the BAL trough structure discussed thus far have been mainly qualitative in nature. In order to evaluate the reality of the two subtroughs quantitatively, we measured the residual intensities at several outflow velocities in each of the BALQSO spectra. These velocity bins are given in Table 2. Each velocity bin is $1000 \mathrm{~km} \mathrm{~s}^{-1}$ wide, and consists of 5 pixels in the individual spectra. One velocity bin is centered upon each of the subtroughs 1 and 2 , and a third velocity bin represents a "reference" region, between -5800 and $-6800 \mathrm{~km} \mathrm{~s}^{-1}$, which separates subtroughs 1 and 2. After the spectra were rebinned to a common number of Angstroms per pixel (conserving flux), the median residual intensity within each of the three velocity bins was determined for each object. We note here that the choices of bin width and position, which are based upon a perusal of Figure 4, are somewhat arbitrary. Given the uncertainty in the absolute velocities of the BAL troughs, some troughs in some objects may lie very nearby the bin window and still fall outside. Thus, while such a trough might contribute to the double-trough structure, some of this information may be lost in the process of binning. On the other hand, larger windows begin to smear out the residual intensity information.

In the next section we describe two experiments where we compare the median residual intensities within the three velocity bins in two different ways: we compare the residual intensities themselves and the differences in residual intensities. Unfortunately, neither experiment gives unambiguous results. One caution: as Figure $9 a$ and individual BAL spectra show, the residual intensity of typical broad absorption decreases sharply at the lowest outflow velocities, reaches a minimum, and more slowly ramps up towards a value of 1 at higher outflow velocities. In other words, after the onset of broad absorption, the absorption is systematically stronger at lower outflow velocities. One must keep this in mind when making comparisons of BAL residual intensities at various outflow velocities.

\subsubsection{The Kolmogorov-Smirnov Test}

Here we test whether or not the distributions of residual intensities which fall within each of the subtrough velocity bins belong to the same population as that which falls within the reference bin. Large differences in the residual intensity distributions will result in small K-S probabilities. Thus we attempt to confirm statistically that which our eye perceives in Figure 4. A disadvantage of this method (or any method which de- 

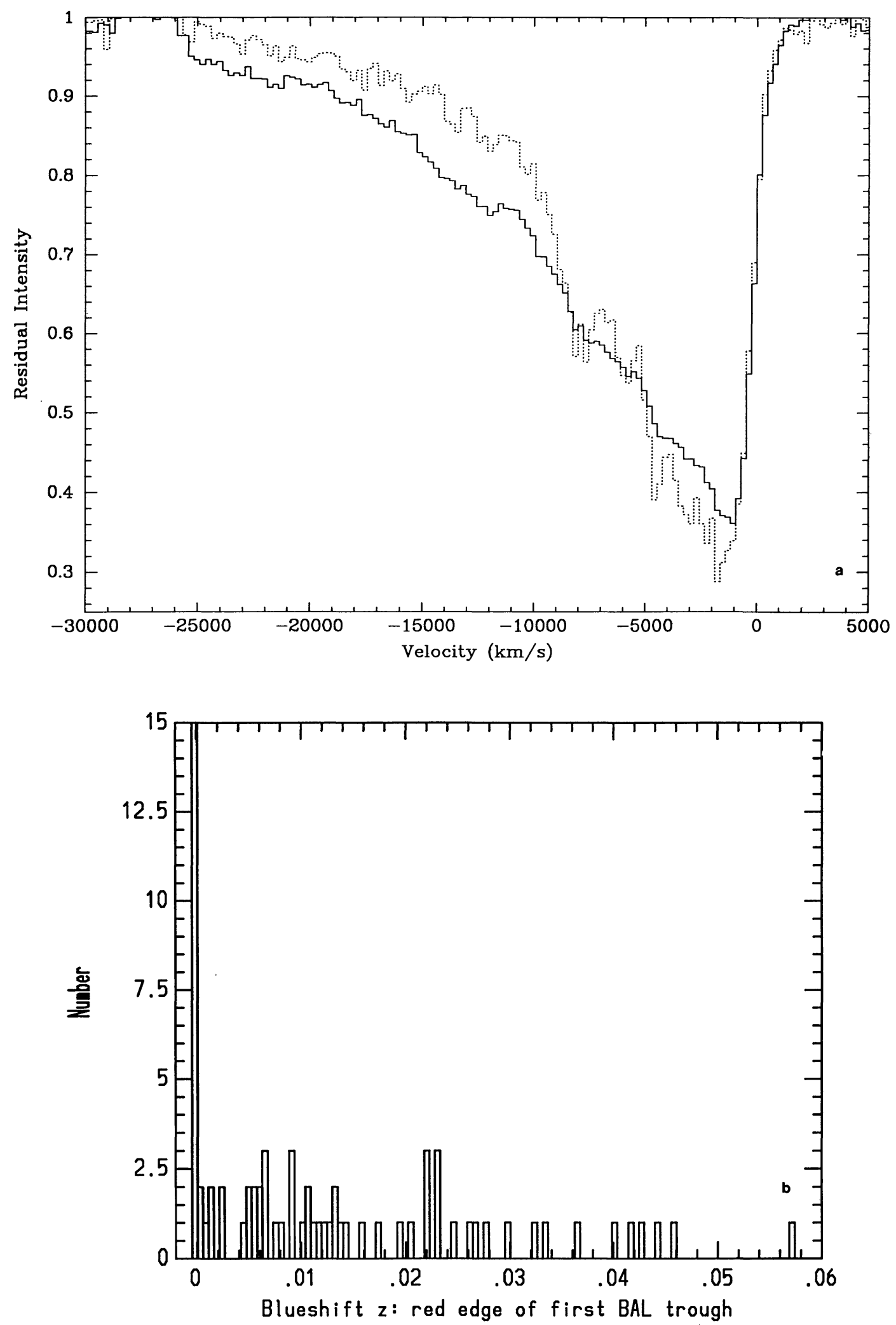

FIG. 9.- (a) Mean ( solid line) and median (dotted line) C IV BAL residual intensity spectra for a sample formed, as described in the text, by shifting all spectra so that the red sides of their first BAL troughs all coincide. Note that the results are nearly featureless, indicating that whatever mechanism is responsible for the origin of the double-trough structure is not tied to the frame of the first broad absorption trough. This figure also illustrates the general smooth falloff in the BAL optical depth as a function of relative velocity shift from the initial trough. $(b)$ Distribution of the blueshifts to the red edge of the first BAL trough (as defined in the text), binned on the width of the double trough features $\left(2500 \mathrm{~km} \mathrm{~s}^{-1}\right)$. 
TABLE 2

RESIDUAL INTENSITY VELOCITY BINS

\begin{tabular}{cc}
\hline $\begin{array}{c}\text { Bin Type } \\
(1)\end{array}$ & $\begin{array}{c}\text { Outflow Velocity Bin } \\
\left(\mathrm{km} \mathrm{s}^{-1}\right) \\
(2)\end{array}$ \\
\hline $\begin{array}{c}\text { Subtrough } 1 \ldots \ldots \\
\text { Reference } \ldots \ldots \ldots\end{array}$ & $4300-5300$ \\
Subtrough $2 \ldots \ldots$ & $5800-6800$ \\
\hline
\end{tabular}

pends solely upon the values of the residual intensity within a velocity bin) is that one cannot distinguish between the BALQSOs whose troughs are distinct and fall within the bins and those whose troughs are just very broad. The latter type would contribute very little to the structure reported in W91. Additionally, this analysis does not distinguish between structure produced by actual double-troughs and that produced by a bimodal velocity distribution of single BAL troughs. Thus, while we can test whether the subtroughs have residual intensities which differ statistically from those in the reference bin, this is not the same as testing whether the double-trough phenomenon within the BALQSO spectra is real.

Nevertheless, the K-S test was conducted on the W91 sample of 34 , the new sample of 38 , the total sample of 72 , and the random- $z$ sample of 72 for comparison. Table 3 lists the onesided K-S probabilities for each subtrough ( 1 and 2 ) velocity bin for each of the samples. We consider K-S probabilities of $1 \%$ and less to represent convincing evidence that the residual intensity distributions are derived from different populations. Probabilities between 1\% and 5\% are suggestive of such. From columns ( 3 ) and (4) of Table 3 it can be seen that none of the samples have K-S probabilities at less than the $5 \%$ level for either subtrough.

\subsubsection{A Monte Carlo Simulation of the Double Trough}

Here, for each BALQSO, we take all possible differences between the values of the median residual intensity within each of the three velocity bins representing the two subtroughs and the reference bin. An important aspect of this scheme is that the amplitude and sign of the differences both provide information. It is the sign which determines the phase, i.e., whether there is a local trough, peak, or a continuation of either (for differences very near zero) at those outflow velocities. This method then largely avoids the ambiguities associated with the use of the residual intensities alone, as described in the previous section. However, the algorithm we apply to these differences to measure the "double trough-ness" (see below) still does not distinguish between a single very deep trough at the position of one of the subtroughs, with the other two velocity bins having residual intensities near 1.0, and a moderately strong double-trough signature. There are perhaps six or seven objects in the total sample where this ambiguity might be present. Other algorithms involving residual intensity differences were tried, but the simple algorithm described below yielded the least ambiguous results.

The following Monte Carlo simulation was performed to test how frequently the depth of the double trough structure in our mean residual intensity spectrum is exceeded by a random arrangement of residual intensity differences among the three velocity bins. The array of 6 by $N$ residual intensity differences ( $N=$ number of BALQSOs in the sample) is randomly sampled twice for each member of the BALQSO sample. The mean of the two randomly chosen residual intensity differences is taken for each member, and the mean of these means across all members gives the statistic $\langle D\rangle_{\text {random }}$. This number is then compared to a number $\langle D\rangle$ calculated in the same way, but using two particular residual intensity differences $\left(R-T_{1}\right)$ and $\left(R-T_{2}\right)$ :

$$
\langle D\rangle=(2 N)^{-1} \Sigma\left[\left(R-T_{1}\right)+\left(R-T_{2}\right)\right],
$$

where $T_{1}$ and $T_{2}$ are the values of the median residual intensities within the velocity bins representing subtroughs 1 and $2, R$ is the median residual intensity within the reference bin, and $N$ is the number of BALQSOs within the sample. Thus $\left(R-T_{1}\right)$ and $\left(R-T_{2}\right)$ are the "unscrambled" residual intensity differences, and these differences are summed up for all $N$ and normalized by twice $N$. Note that this calculation is the same as that described above for two residual intensity differences chosen at random.

The quantity $\langle D\rangle$ can range over \pm 1 and is in some sense a measure of the contrast between the double-trough structure and the large-scale BAL envelope. A value of $\langle D\rangle$ near +1 will result from those BALQSOs with $\mathrm{C}$ IV troughs which show a very strong double-trough signature at the two subtrough velocities, while a value near -1 will result from those BALQSOs having a single very deep trough centered on the reference bin. A value near zero is representative of a smooth trough through the three velocity bins (i.e., the BAL has constant slope through all three velocity bins).

The process of determining $\langle D\rangle_{\text {random }}$ from two random selections of residual intensity differences and comparing to $\langle D\rangle$ was repeated 10,000 times. The total number of instances in which a $\langle D\rangle_{\text {random }}$ calculated from a random selection of residual intensity differences exceeds $\langle D\rangle$, normalized by 10,000 , gives the probability that a double-trough structure having at least the contrast observed in the sample mean residual intensity spectrum could arise by chance. These probabilities and the values of $\langle D\rangle$ are given in Table 4 for the same samples considered in the K-S test. The probabilities derived for the W91 and the total samples are at the $2 \%$ and $5 \%$ levels, respec-

TABLE 3

THE KOLMOGOROV-SMIRNOV TEST ON FOUR SAMPLES

\begin{tabular}{cccc}
\hline \hline & & \multicolumn{2}{c}{ K-S PrOBABILITIES $\times 100 \%$} \\
\cline { 3 - 4 } $\begin{array}{c}\text { SAMPLE } \\
\text { (1) }\end{array}$ & $\begin{array}{c}\text { SizE } \\
(2)\end{array}$ & $\begin{array}{c}\text { Subtrough 1 } \\
(3)\end{array}$ & $\begin{array}{c}\text { Subtrough 2 } \\
(4)\end{array}$ \\
\hline Weymann et al. $\ldots \ldots \ldots$ & 34 & 7.67 & 13.0 \\
New sample $\ldots \ldots \ldots \ldots$. & 38 & 75.1 & 35.1 \\
Total sample $\ldots \ldots \ldots \ldots$ & 72 & 30.0 & 16.8 \\
Random- $z$ sample $\ldots \ldots$. & 72 & 85.9 & 85.9 \\
\hline
\end{tabular}

NOTE.-The entries in the table give the probability in percentages that the K-S statistic would be exceeded by chance if the subtrough and reference bin residual intensity distributions were drawn from the same parent population. 
TABLE 4

Monte Carlo Simulations of the Double Trough FOR FOUR SAMPLES

\begin{tabular}{ccc}
\hline \hline $\begin{array}{c}\text { BALQSO Sample } \\
(1)\end{array}$ & $\begin{array}{c}\langle D\rangle \\
(2)\end{array}$ & $\begin{array}{c}\text { Monte Carlo } \\
\text { Probability } \times 100 \% \\
(3)\end{array}$ \\
\hline Weymann et al. $\ldots \ldots \ldots$ & 0.0988 & 2.03 \\
New sample $\ldots \ldots \ldots \ldots$ & 0.0199 & 35.4 \\
Total sample $\ldots \ldots \ldots \ldots$ & 0.0553 & 5.02 \\
Random- $z$ sample $\ldots \ldots$. & -0.0318 & 88.2 \\
\hline
\end{tabular}

NOTE.-The entries in the last column show the percentage of 10,000 trials in which the $\langle D\rangle$ calculated using random selections of velocity bin residual intensity differences was greater than the value of $\langle D\rangle$ for the sample, given in col. (2).

tively. The same experiment applied to the random- $z$ sample resulted in a small (and negative) $\langle D\rangle(-0.0318)$ and a large probability, as one might expect. We conclude that the Monte Carlo simulation, which takes advantage of a better indicator of "double-troughness", suggests, but does not conclusively establish, that the C IV BAL structure is telling us something about the nature of the BAL cloud acceleration mechanism in at least some BALQSOs.

\subsection{A Comparison of the Properties of the Double-Trough $B A L Q S O$ s and those without}

It is apparent that the majority of BALQSOs do not produce double troughs near -4700 and $-10,500 \mathrm{~km} \mathrm{~s}^{-1}$, such as those which appear in Figure $8 a$. It may be that physical conditions within the BAL regions, which are very likely tied to the workings of the central engine, dictate whether or not double troughs are formed. If there were some other property (e.g., emission line or continuum) of the double-trough sample which separated them from those which did not produce the double troughs and which could have some causal relationship, this might lend more credence to the reality of the double-trough phenomenon. W91 found that the so-called lowionization BALQSOs have very different emission line and continuum properties from those with only high-ionization BALs. Thus these were removed from both samples (one from the double-trough sample and 12 from the other). The LCO spectra taken in 1991 October were also excluded from this non-double-trough sample (there were none of these in the double-trough sample), as their wavelength coverage is small and their relative fluxes much more uncertain shortward of the $\mathrm{C}$ IV trough. This left 31 objects of the original 56 in the nondouble-trough sample, and 15 of 16 in the double-trough sample.

In Figure 10 we show the mean spectra of the adjusted double-trough and non-double-trough samples. Their continua are identical. Other than the differences expected from their different outflow velocity distributions in broad absorption, their emission lines are also nearly identical. The largest difference is in the strength of the $\operatorname{Ly} \alpha$ emission, and this is explained by the much stronger (i.e., more concentrated) absorption between $-4000 \mathrm{~km} \mathrm{~s}^{-1}$ and $-11,000 \mathrm{~km} \mathrm{~s}^{-1}$ in the double-trough sample which absorbs this emission. The heavier, on average, absorption of the $\operatorname{Ly} \alpha$ emission in the double-trough sample would be expected if that process is involved in the formation of the double troughs $(\S 5)$. Note, however, that both samples strongly absorb the broad emission line Ly $\alpha$. The non-double-trough sample also shows stronger absorption at the lowest outflow velocities, and this probably accounts for the slightly weaker $\mathrm{C}$ IV and $\mathrm{N} v$ broad emission peaks in this sample. We find no significant differences in the continuum and emission-line properties between these two samples. Comparisons based upon other non-BAL trough properties, such as radio and X-ray, are inconclusive because the available data are scanty.

Summarizing: although the evidence for the reality of the double trough is not conclusive, it is suggestive. Evidently, a majority of BALQSOs do not produce double-trough BALs, but a substantial fraction (22\%) of the 72 studied here do. Another three BALQSOs in this study show double troughs at outflow velocities other than those observed in the difference spectrum of W91, and three BALQSOs belonging to the double-trough sample have at least one other set of double troughs occurring at other outflow velocities. It is possible that the current number of BALQSO spectra is still not adequate to address the statistical nature of the problem. The differences between Figures 4 and 5 (the old and new samples) suggest that this may be the case. However, under the assumption that the double-trough phenomenon is real, we discuss possible origins in the next section.

\section{POSSIBLE LINE-LOCKING ORIGINS OF ABSORPTION- TROUGH STRUCTURE}

The theoretical implications of the apparent double-trough structure in C IV absorption are potentially far-reaching. Most intriguingly, the velocity separation of the troughs, almost identical to the velocity-space separation between Ly $\alpha$ and the $\mathrm{N} v$ resonance doublet, suggests that line-locking and radiation pressure play an important role in BAL cloud acceleration. Bearing in mind that we have not yet demonstrated conclusively that the double troughs are more than mere statistical fluctuations, we forge ahead in this section and briefly explore some of their implications in the hope of motivating further observational and theoretical work on this subject. We begin by discussing several general mechanisms for line-locking and then focus on the specific case of $\mathrm{Ly} \alpha-\mathrm{N} v$ line locking in QSO outflows.

\subsection{Line-Locking Mechanisms}

The term "line-locking" refers generically to nonlocal coupling processes in radiation-hydrodynamical flows in which photons resonant with one transition (at frequency $\nu_{1}$ ) in one part of a flow Doppler shift into resonance with another transition $\left(\right.$ at $\left.\nu_{2}\right)$ in another part of the flow. In an expanding flow where $d(\boldsymbol{v} \cdot \hat{\boldsymbol{r}}) / d \boldsymbol{r}>0$ along all lines of sight, radiation traveling through the medium progressively redshifts with respect to the local comoving reference frame. Photons of frequency $\nu_{1}>$ $\nu_{2}$ created at some point $\boldsymbol{r}_{1}$ in the flow then exert a pressure on gas along the surface defined by $\boldsymbol{r}_{2}$ such that

$$
\left[v\left(r_{2}\right)-v\left(r_{1}\right)\right] \cdot \frac{r_{2}-r_{1}}{\left|r_{2}-r_{1}\right|}=\frac{\nu_{1}-\nu_{2}}{\nu_{1}} c .
$$




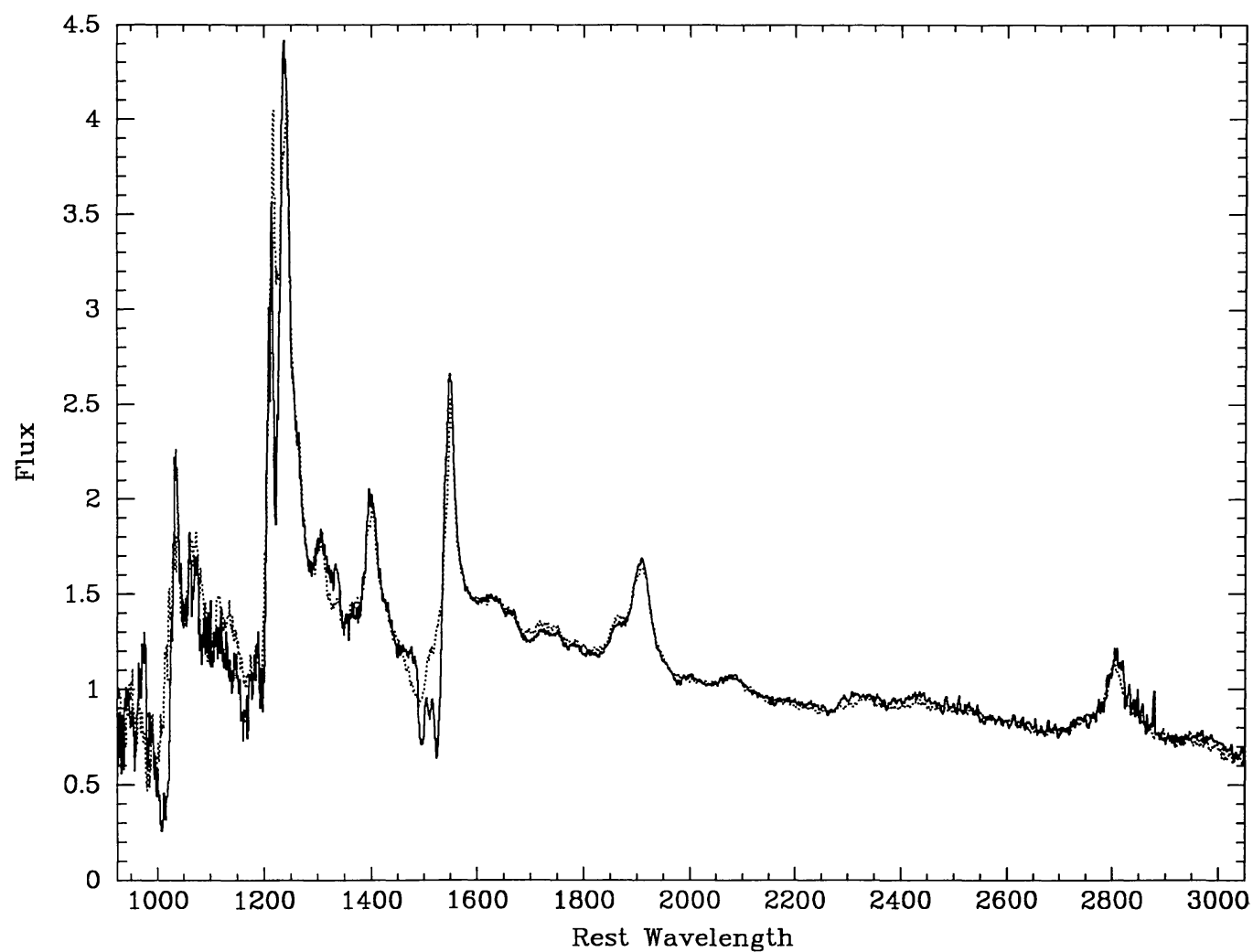

FIG. 10.-Mean emission-line and continuum spectra of the double-trough (solid line) and non-double-trough (dotted line) samples. Contributions from the 1991 LCO BALQSOs and the Mg II BALQSOs have been removed from each. Note, the close similarities in the continuum and emission-line properties.

In a contracting flow $[d(\boldsymbol{v} \cdot \hat{\boldsymbol{r}}) / d \boldsymbol{r}<0]$, all points are blueshifted with respect to one other, and the frequency relationship for line locking reverses $\left(\nu_{1}<\nu_{2}\right)$. If the flow dynamics at $\boldsymbol{r}_{2}$ are sensitive to these radiation forces, line-locking effects can develop, particularly when such alterations in the radiation force cause the net acceleration to vanish (Scargle, Caroff, \& Noerdlinger 1970; Mushotzky, Solomon, \& Strittmatter 1972; Scargle 1973).

Absorption-line locking can occur when line-of-sight absorption in one transition blocks photons that would otherwise be absorbed by a second, decreasing the radiation pressure associated with the second transition. Radiation of frequency $\nu_{0}>\nu_{1}>\nu_{2}$ from a source at $\boldsymbol{r}_{0}$ in an expanding flow can be absorbed on two different surfaces corresponding to $\nu_{1}$ and $\nu_{2}$. Along any line of sight where $d(\boldsymbol{v} \cdot \hat{\boldsymbol{r}}) / d \boldsymbol{r}$ is always positive, the surface absorbing at $\nu_{1}$ will lie closer to the continuum source than the one absorbing at $\nu_{2}$, and absorption at the nearer surface will reduce the radiation pressure on the farther one. Again, the ordering of the frequencies must be reversed for a contracting flow.

To illustrate the effects of radiation-pressure reduction on an absorption-line spectrum, let us consider the simplistic case of many identical discrete absorbing clouds embedded in a steady radial outflow accelerating according to the velocity law $v(r)$. If the rate at which clouds flow outward is constant, the number of clouds in any velocity interval $\Delta v$ is proportional to $(\Delta v / v)(d r / d v)$. A decrease in the acceleration localized at velocity $v$ leads to an excess of absorbers at $v$, and a localized increase in the acceleration leads to a deficit in absorption.
Because linear fluctuations in the acceleration produce linear fluctuations in the number of absorbers, the fractional change in the number of absorbers at $v$ is similar to the fractional change in the overall acceleration. At velocities where the acceleration drops nearly to zero, large accumulations of absorbing clouds can produce deep absorption features.

Line-locking, narrowly defined, occurs when shadowing of photons at $\nu_{2}$ by a transition at $\nu_{1}$ causes the cloud acceleration to vanish. This paper employs a broader definition of the term which includes the qualitatively similar but smaller amplitude effects that arise from modest decreases in the acceleration. When a transition at $\nu_{1}$ shadows another at $\nu_{2}$ enough to perturb the acceleration significantly, a weak absorption linelocking feature can appear. If radiation pressure dominates all other forces, line-locking then affects the absorption profiles only insofar as the radiation force on the transition at $\nu_{2}$ contributes to the overall acceleration.

Locking of resonance lines with ionization edges was once thought to influence QSO absorption-line systems (Scargle et al. 1970; Burbidge \& Burbidge 1975). In this scheme, a continuum radiation field that cuts off abruptly above the Lyman limit or some other convenient edge accelerates absorbing clouds optically thin in the continuum but optically thick in resonance lines. If only a few lines contribute to the overall acceleration, the radiation force on a given cloud drops sharply each time an absorbing line shifts past the ionization edge. Features in the velocity-space distribution of clouds would then be expected at the velocity shifts given by edge-line resonances. However, QSO absorption-line systems tend not to 
show absorption features unusually frequently at the expected velocity resonances (e.g., Weymann, Carswell, \& Smith 1981), and the evidence is now overwhelming that the displaced narrow lines are cosmologically distant.

In general, numerous resonance lines $(\gtrsim 13)$ can and probably do contribute to the total radiation force on a BAL cloud (Korista et al. 1992). Elimination of the radiation force on any one of these through absorption line-locking alters the overall radiation force by a relatively small amount. According to the simple model outlined above, such small changes in the radiation force should produce only small fluctuations in the absorption-line profiles, but when the total outward force on the clouds, from radiation and other kinds of pressure, just slightly exceeds the total inward force, from gravity and possibly drag against a more slowly moving confining medium, a small decrease in the total radiation force can produce a large relative decrease in the net force (Scargle 1973). When subtle balancing between forces allows absorption line-locking to reduce the net acceleration sharply, clouds downstream from a deep absorption trough at velocity $v$ will stagnate at a velocity $v$ $+c\left(1-\nu_{2} / \nu_{1}\right)$, giving a second trough resonant with the first. Additional resonant troughs with the same velocity separation can develop further downstream as long as the force balancing is favorable.

A similar kind of absorption-line locking, in which sets of locked clouds accelerate in unison, can operate under less delicately tuned conditions (Braun \& Milgrom 1989). For instance, consider a system of clouds whose acceleration, in the absence of line-locking, is a monotonically increasing function of radius. The relative velocity between two clouds at different radii along the same line of sight thus increases until the two clouds enter into an absorption-line locking resonance. If resonance-line shadowing can potentially reduce the acceleration of the downstream cloud below the acceleration of the upstream cloud, the two clouds can become stably locked together in velocity space. When the initial velocity separation is smaller than the resonant value, the downstream cloud accelerates into the shadowed interval of velocity space where the radiation force on it decreases until the accelerations of the two clouds become equal. The clouds subsequently accelerate together while maintaining the same velocity-space separation, slightly smaller than the precise resonance value for maximum shadowing. This type of locking, described more generally by Braun \& Milgrom (1989), might govern the observed locking of resonance-line doublets in some BALQSOs (Turnshek et al. 1980; Turnshek et al. 1984; Foltz et al. 1987). We emphasize that the resonance-line doublet line-locking is not the phenomenon under discussion here.

\section{2. $\mathrm{Ly} \alpha-\mathrm{N} \mathrm{v}$ Line-Locking in BALQSOS}

The mean C IV line profile of our BALQSO sample shows prominent minima at radial velocities of $\sim-4700$ and $\sim-10,500 \mathrm{~km} \mathrm{~s}^{-1}$. Their relative separation of $\sim 5800 \mathrm{~km} \mathrm{~s}^{-1}$ corresponds closely to the velocity-space interval between Ly $\alpha$ $\lambda 1215.7$ and the $\mathrm{N} \mathrm{v}$ resonance-line doublet at $\lambda \lambda 1238.8$, 1242.2 , suggesting that some kind of line-locking mechanism might be important in determining the dynamics of BAL outflows. Because the resonant shift is between absorption troughs, absorption-line locking seems most likely.

The fact that the two subtroughs appear in a mean BALQSO spectrum is important for two reasons. First, it excludes a Braun-Milgrom type of mechanism in which the velocities of the locked systems would not be correlated from object to object. (Figs. $9 a$ and $9 b$ at the end of $\S 4.3$ supply additional evidence against relative (outflow) velocity line locking.) Second, it indicates that individual absorption-line systems are sensitive to the systemic velocity of the host QSO. Thus, velocity information in the radiation field propagating outward from the QSO nucleus must be passing from low-velocity regions to higher velocity regions, implying an accelerating outflow in which $\mathrm{Ly} \alpha$ radiative transfer at small radii affects the radiation pressure on $\mathrm{N} \mathrm{v}$ at larger radii (i.e., expansion implies $\nu_{1}>\nu_{2}$ ).

Each of the alleged line-locking subtroughs in the mean C IV spectrum drops $10 \%$ or so lower than the rest of the absorption profile. Because fluctuations of this size approximate the relative contribution of $\mathrm{N} \mathrm{v}$ to the total radiation force, it is natural to suppose that simple Ly $\alpha$ shadowing of $\mathrm{N} \mathbf{v}$ might actually generate the trough-trough resonance. However, we have already shown that the line-locking signature in the mean spectrum is produced primarily by large-amplitude features in a small subset $(22 \%)$ of the entire sample (see Fig. $8 a$ ). Such deep features cannot be produced by simple absorption-line locking; they require either delicate force balancing in which the net acceleration can vanish or some kind of nonlinear amplification mechanism which dramatically increases the absorption in response to small changes in the total radiation pressure.

Let us first consider delicate force balancing. Radiation pressure certainly contributes to the outward acceleration of a cloud, with each resonance line providing $\sim 10 \%$ or so of the total radiation force. Gravity exerts an opposing but unimportant inward force; the radiation pressure on BAL clouds is likely to overwhelm the pull of gravity (e.g., Begelman, DeKool, \& Sikora 1991). More importantly, drag forces will oppose radiative acceleration as photon pressure pushes cool clouds through a hotter confining medium (e.g., Begelman et al. 1991). Clouds driven through a hot medium soon achieve terminal relative velocities that depend on the total radiation force. The radial velocity $v$ of such a cloud equals the flow velocity of the hot gas $v_{h}$ plus its terminal velocity $v_{t}$ with respect to the background flow, and typically $v_{t} \ll v_{h}$ (Begelman et al. 1991). In evaluating the fluctuations in acceleration, it will be useful to consider $v_{t}$ and $v_{h}$ separately.

Although the inward and outward forces that determine $v_{t}$ closely balance, the inward force always adjusts itself to the outward force. Reductions in the radiation pressure merely reduce the equilibrium value of $v_{t}$, which decreases as a cloud moves into a shadowed interval of velocity space and increases as the cloud leaves. The relative change in acceleration during the passage is $\sim d v_{t} / d v_{h}$. If shadowing reduces the radiation force by a factor $f_{\mathrm{sh}} \sim 0.1$ over the velocity interval $\delta v$, the relative depth of the responding absorption feature will be $\sim f_{\mathrm{sh}} v_{t} / \delta v$. Deep features are possible if the shadowing feature has an abrupt edge $\left(\delta v \ll v_{t}\right)$, but they would then be quite narrow, with equivalent widths in velocity space of only $\sim f_{\mathrm{sh}} v_{t}$. The force balance that sets $v_{t}$ is therefore unlikely to yield significant line-locking features.

The role of absorption-line shadowing in determining $v_{h}$ is more difficult to assess. A proper treatment of possible linelocking effects on $v_{h}$ would begin with a global model for BAL 
cloud acceleration in which radiation pressure on embedded absorbing clouds drives the background confining flow. The delicate force balancing needed to form a deep absorption feature would have to be between gravitation on the entire flow, hot plus cold gas, and radiation pressure on the cool clouds. Models of this type require a very low-density, high-temperature confining medium (Weymann, Turnshek, \& Christiansen 1985); otherwise, the inertia of the confining medium would prohibit acceleration of the BAL clouds to the observed velocities. X-rays alone cannot heat the gas to high enough temperatures $\left(\gg 10^{8} \mathrm{~K}\right)$; some type of relativistic heating mechanism (e.g., Begelman et al. 1991) is needed. Further investigation along these lines awaits a self-consistent model for high-temperature radiation-driven outflows.

Nonlinear amplification of absorption features initiated by small radiation-pressure adjustments could conceivably develop in several different ways, but again the viability of these mechanisms is difficult to evaluate theoretically while we still lack an adequate model for cloud acceleration. For instance, if the ionization balance in an absorbing cloud is very sensitive to the cloud pressure, small increases in absorption-line shadowing might raise the internal cloud pressure enough for a large change in the ionization balance to occur. Mechanisms of this type can be evaluated observationally through comparisons of absorption troughs from species sensitive to various ionization parameters. Amplification might also ensue from small variations in the radiation pressure if these variations produce large changes in cloud stability. Shadowed clouds might then remain more stable longer, persist at larger thicknesses, and thus absorb more thoroughly. Clearly, theoretical treatments of higher order dynamical problems such as these first require a solution of the zeroth-order problem of cloud acceleration.

Finally, it is possible that the absorption minima arise from a complex combination of absorption and emission line-locking effects. Consider the history of an absorption-line cloud, formed at zero velocity near the nucleus, that manages to remain stable as it accelerates to high velocities. When close to the nucleus, the cloud sees an unobscured continuum plus emission-line spectrum. Because absorption lines in low-velocity clouds absorb photons near the peaks of the emission lines, the cloud experiences radiation forces that begin large and decrease gradually as the cloud accelerates down the blue wings of the emission lines. A population of such clouds would bunch more and more tightly in velocity space, absorbing more deeply at several thousand $\mathrm{km} \mathrm{s}^{-1}$ than at zero velocity. As the cloud velocity shifts toward the $\operatorname{Ly} \alpha-\mathrm{N} v$ velocity resonance, the radiation pressure on $\mathrm{N} v$ becomes increasingly more important as the cloud climbs the red wing of $\operatorname{Ly} \alpha$, reversing the bunching and perhaps creating the first minimum in the absorption profile. At higher velocities, $\mathrm{N} \mathrm{v}$ will cross the peak and descend down the blue wing of $\operatorname{Ly} \alpha$. The radiation force on $\mathrm{N} v$ associated with the blue wing will be diminished by Ly $\alpha$-absorbing clouds closer to the source, reaching a minimum when the $\mathrm{N} v$ line shifts to the bottom of the first Ly $\alpha$ trough. The depth of the second trough, due to this second dropoff in acceleration, will reflect the degree to which Ly $\alpha$ pressure on $\mathrm{N} \mathrm{v}$ has come to dominate the total outward force. Scenarios such as this are unsatisfyingly complex, but they will need to be worked out in greater detail if the resonant subtroughs prove unquestionably to be real. No simple mechanism involving radiation pressure alone can create deep subtroughs through shadowing of a single line.

\section{SUMMARY}

We have investigated whether the double trough structure in the mean C IV BAL trough reported by W91 is real or due to statistical fluctuations of BAL troughs occurring over random outflow velocities in a limited sample. To do this we analyzed a sample of 72 BALQSOs with C IV BAL troughs, doubling the sample size of W91. Although the evidence for the reality of the double troughs is not conclusive, it is suggestive. We believe that a still larger sample is needed to properly answer the question above. On the assumption that the structure is real, we discussed possible scenarios for the origin of the double troughs involving the line-locking of $\mathrm{Ly} \alpha-\mathrm{N} \mathrm{v}$. We showed that no simple mechanism involving radiation pressure alone can create the deep double troughs through the shadowing of $\mathrm{N} \mathrm{V}$ $\lambda 1240$ by Ly $\alpha$. More complicated amplification mechanisms may be necessary. The possible reality of the double-trough signature has the following important implications to our understanding of the BAL phenomenon: (1) The occurrence of broad absorption is somehow tied to an absolute velocity frame which is the same from quasar to quasar. (2) It follows then that the outflowing material within the BAL region is accelerating. (3) If line locking is the mechanism behind the formation of the double troughs, then radiation pressure plays an important role in the dynamics of the BAL region.

K.T.K., R.J.W., and S.L.M. wish to acknowledge support through the National Science Foundation grant AST 90-05117 and NASA grant NAG 5-1859. G.M.V. acknowledges support through the NASA grants NAGW 2142 and NAGW 2144. We also thank Mitch Begelman for helpful conversations.

\section{REFERENCES}

Begelman, M. C., De Kool, M., \& Sikora, M. 1991, ApJ, 382, 416

Braun, E., \& Milgrom, M. 1989, ApJ, 342, 100 1990, ApJ, 349, L35

Burbidge, E. M., \& Burbidge, G. R. 1975, ApJ, 202, 287

Corbin, M. R. 1990, ApJ, 357, 346

Espey, B. R., Carswell, R. F., Bailey, J. A., Smith, M. G., \& Ward, M. J. 1989, ApJ, 342, 666

Foltz, C. B., Weymann, R. J., Morris, S. L., \& Turnshek, D. A. 1987, ApJ, 317,450

Gaskell, C. M. 1982, ApJ, 263, 79

Korista, K. T., et al. 1992, ApJ, 401, 529

Mushotzky, R. F., Solomon, P. M., \& Strittmatter, P. A. 1972, ApJ, 174, 7

Scargle, J. D. 1973, ApJ, 179, 705

Scargle, J. D., Caroff, L. J., \& Noerdlinger, P. D. 1970, ApJ, 161, L115

Stocke, J. T., Morris, S. L., Weymann, R. J., \& Foltz, C. B. 1992, ApJ, 396, 487
Stone, R. P. S., \& Baldwin, J. A. 1983, MNRAS, 204, 347

Turnshek, D. A., Foltz, C. B., Grillmair, C. J., \& Weymann, R. J. 1988 , ApJ, 325, 651

Turnshek, D. A., Weymann, R. J., Carswell, R. F., \& Smith, M. G. 1984, ApJ, 277, 51

Turnshek, D. A., Weymann, R. J., Liebert, J. W., Williams, R. E., \& Strittmatter, P. A. 1980, ApJ, 238, 488

Weymann, R. J., Carswell, R. F., \& Smith, M. G. 1981, ARA\&A, 19, 41 Weymann, R. J., Turnshek, D. A., \& Christiansen, W. A. 1985, in Astrophysics of Active Galaxies and Quasi-Stellar Objects, ed. J. S. Miller (Mill Valley: University Science Books), 333

Weymann, R. J., Morris, S. L., Foltz, C. B., \& Hewett, P. C. 1991, ApJ, 373,23 (W91) 